\title{
SOME REGULARITY RESULTS FOR MINIMAL CRYSTALS
}

\author{
L. Ambrosio ${ }^{1}$, M. Novaga $^{2}$ And E. Paolini ${ }^{1}$
}

\begin{abstract}
We introduce an intrinsic notion of perimeter for subsets of a general Minkowski space (i.e. a finite dimensional Banach space in which the norm is not required to be even). We prove that this notion of perimeter is equivalent to the usual definition of surface energy for crystals and we study the regularity properties of the minimizers and the quasi-minimizers of perimeter. In the two-dimensional case we obtain optimal regularity results: apart from a singular set (which is $\mathcal{H}^{1}$-negligible and is empty when the unit ball is neither a triangle nor a quadrilateral), we find that quasi-minimizers can be locally parameterized by means of a bi-lipschitz curve, while sets with prescribed bounded curvature are, locally, lipschitz graphs.
\end{abstract}

Mathematics Subject Classification. 49J45, 49Q20.

Received October 4, 2001.

\section{INTRODUCTION}

In this paper we consider the minimizers and the quasi-minimizers of the functional

$$
E \mapsto P(E):=\int_{\partial^{*} E} \varphi\left(\nu_{E}(x)\right) \mathrm{d} \mathcal{H}^{n-1}(x),
$$

defined for a set $E \subset \mathbb{R}^{n}$ with finite perimeter. In (1) $\partial^{*} E$ represents the reduced boundary of the set $E$ (i.e. the points of the boundary where a generalized normal vector is defined), $\nu_{E}(x)$ denotes the exterior unit normal vector to $E$ at $x \in \partial^{*} E$, and $\varphi: \mathbb{R}^{n} \rightarrow[0, \infty[$ is a positively 1-homogeneous convex function. We observe that, when the function $\varphi$ is even (hence 1-homogeneous), the functional (1) provides an intrinsic notion of perimeter when one endowes $\mathbb{R}^{n}$ with a suitable Banach structure related to $\varphi$ (see Th. 2.7).

The minima of (1) have been widely studied in the literature $[3,9,26]$, in particular it is well known $[3,10]$ that, whenever $\varphi$ is smooth and uniformly elliptic out of the origin, the minima are hyper-surfaces of class $\mathcal{C}^{1, \alpha}$ out of a "small" singular set. Here, we are mainly interested in the case of general convex functions $\varphi$. In this situation it is quite easy to provide examples of minima which are locally the graph of a lipschitz, but not differentiable, function. Moreover, the boundary of these sets may have singular points (i.e. points where $\partial E$ is not a manifold) also in two dimensions. These examples have been studied in particular by Taylor and Cahn [29], Morgan [23]), which have also classified the singular cones which are minimal for the functional

\footnotetext{
Keywords and phrases: Quasi-minimal sets, Wulff shape, crystalline norm.

1 Scuola Normale Superiore, Piazza dei Cavalieri 7, 56126 Pisa, Italy; e-mail: ambrosio@emath.ethz.ch

2 Dipartimento di Matematica, Università di Pisa, via F. Buonarroti 2, 56126 Pisa, Italy.
} 
in (1). As has been pointed out by Taylor in [26], the study of such minimal sets is closely related to problems arising in material science and phase transitions in an anisotropic environment.

We introduce a class of sets which are $\omega$-minimal for (1) (see Def. 3.1), including the minima for

$$
E \mapsto P(E)+\int_{E} H(x) \mathrm{d} x
$$

with $H \in L^{p}\left(\mathbb{R}^{n}\right), p \geq n$. In two dimensions, i.e. for curves in $\mathbb{R}^{2}$, we are able to prove that the boundaries of these $\omega$-minima are parameterizable by means of a bi-lipschitz map in a neighbourhood of almost any point of the boundary, i.e. the set of singular points has zero measure with respect to $\mathcal{H}^{1}$ (Th. 6.14). We also show that this regularity result is optimal. In the particular case of minima of (2), when $H \in L^{\infty}\left(\mathbb{R}^{2}\right)$, we use the additional regularizing effect of the volume integral to improve the result by showing that the boundary of a minimizer is the graph of a lipschitz function out of a singular set of zero $\mathcal{H}^{1}$-measure (see Prop. 6.5 and Th. 6.19). When the intrinsic unit ball $\{x: \varphi(x) \leq 1\}$ is neither a triangle nor a quadrilateral, these regularity results can be improved by showing that the singular set is actually empty (see Th. 6.18). We point out that a regularity result analogous to Proposition 6.5 has been obtained by Morgan et al. [24], in the case of clusters with prescribed volume which minimize (1). The techniques used are similar: comparing a minimizing curve with the segment having the same extremal points. On the other hand, the idea of the proof of Theorem 6.19 is to compute suitable small variations of the functional (2).

The case of dimension greater than two is still open and deserves further investigation. Surprisingly, the usual techniques (i.e. getting the regularity of the boundary from the decay of a suitable notion of excess) seem to fail in this situation (see the second example in Sect. 7).

The plan of the paper is the following:

In Section 2 we fix the intrinsic notation that we will use throughout the paper. In particular, we interpret the functional $P(\cdot)$ in $(1)$ as a kind of perimeter in $\mathbb{R}^{n}$ equipped with a suitable distance related to $\varphi$. In the case of an even function $\varphi$, a definition of perimeter equivalent to ours has been given and developed in [4].

In Section 3 we give the definition of $\omega$-minimal set, which extends the notion of minimizer for (2). Moreover, we prove that compactness and density estimates hold for this class of sets (see Prop. 3.2, Prop. 3.3 and Prop. 3.5).

In Section 4 we introduce the notion of excess, that is a quantity which measures the "distance" of the set from being flat in a given ball. In Proposition 4.6 we prove that the boundary of an $\omega$-minimal set coincides with the graph of a lipschitz function up to a set whose $(n-1)$-Hausdorff measure is controlled by the excess.

In Section 5 we prove that, under the assumption of uniform convexity of the unit ball of the dual space, a polynomial decay of the excess implies the $\mathcal{C}^{1, \alpha}$-regularity of the boundary of $\omega$-minimal sets.

In Section 6 we consider the case when the ambient space is two-dimensional. In particular we show a decay result for the excess (Lem. 6.2) which implies the $\mathcal{C}^{1, \alpha}$-regularity for $\omega$-minimizers under the convexity assumption of Section 5. We also prove (Th. 6.14) the (local) bi-lipschitz regularity, in the sense of parameterizations, for $\omega$-minimizers in two dimensions out of a singular set of zero $\mathcal{H}^{1}$-measure. In Section 6.4 we show that, when the unit ball of the ambient space is nor a triangle neither a quadrilateral, the set of singular points of the boundary of an $\omega$-minimizer is empty. In Section 6.5 we prove the lipschitz regularity, in the sense of graphs, for the minima of (2), under very general assumption on the shape of the unit ball and on the function $H$ (Th. 6.19).

In Section 7 we provide an example of $\omega$-minimizer in two dimensions which is not (locally) the graph of a lipschitz function, and so we show that Theorem 6.14 is optimal. Moreover, we give an example of global minima for (1) in $\mathbb{R}^{3}$ for which no excess decay property holds. This suggests that, in dimension greater than two, different techniques are needed in order to get a regularity result (if any). 


\section{Preliminary notions}

\subsection{Geometric structure}

Let $X$ be a real $n$-dimensional vector space. We say that a function $\|\cdot\|: X \rightarrow \mathbb{R}$ is a general norm if it satisfies

$$
\begin{array}{cc}
\|x+y\| \leq\|x\|+\|y\|, & \forall x, y \in X, \\
\|\lambda x\|=\lambda\|x\|, & \forall x \in X, \forall \lambda \geq 0, \\
\|x\|=0 \Longleftrightarrow x=0, & \forall x \in X .
\end{array}
$$

We say that $\|\cdot\|$ is even if also $\|x\|=\|-x\|$ holds for every $x \in X$ (in this case $\|\cdot\|$ is a norm in the usual sense). The space $X$ endowed with the general norm $\|\cdot\|$ will be called a general Minkowski space.

Let $X^{*}$ be the dual space of $X$, that is the space of linear functionals on $X$. If $v \in X$ and $\xi \in X^{*}$, we denote by $\langle\xi, v\rangle$ the evaluation of the functional $\xi$ on $v$. If $X$ is a general Minkowski space the dual space $X^{*}$ has the natural general Minkowski structure induced by the general norm $\|\cdot\|: X^{*} \rightarrow \mathbb{R}$ defined by

$$
\|\xi\|:=\sup _{x \in X \backslash\{0\}} \frac{\langle\xi, x\rangle}{\|x\|}
$$

(notice that we will use the same symbol $\|\cdot\|$ for the general norm of both $X$ and $X^{*}$, but the elements of $X^{*}$ will always be denoted by Greek letters). For $x \in X$, and $\rho>0$ we let $B_{\rho}(x):=\{y \in X:\|y-x\|<\rho\}$ be the ball centered in $x$ with radius $\rho$, we also let $B_{\rho}:=B_{\rho}(0)$. The balls of $X^{*}$ will be denoted by $B_{\rho}^{*}(\xi)$ and $B_{\rho}^{*}$. In the literature $B_{1}$ is sometimes called Wulff shape and $B_{1}^{*}$ is called Frank diagram.

Let $v \in X \backslash\{0\}$. We define the orthogonal space $v^{\perp} \subset X^{*}$ and the dual face $v^{*} \subset X^{*}$ by

$$
\begin{aligned}
v^{\perp} & :=\left\{\xi \in X^{*}:\langle\xi, v\rangle=0\right\} \\
v^{*} & :=\left\{\xi \in X^{*}:\|\xi\|=\|v\|,\langle\xi, v\rangle=\|\xi\| \cdot\|v\|\right\}
\end{aligned}
$$

Notice that $v^{*}$ and $v^{\perp}$ are closed convex, non empty sets. Moreover $v^{*} /\|v\|$ is the sub-differential of the convex function $\|\cdot\|: X \rightarrow \mathbb{R}$ at the point $v$. In the sequel, we will always identify $X^{* *}$ with $X$ (this is done by the canonical isomorphism, which preserves the general Minkowski structures) so that if $\xi \in X^{*} \backslash\{0\}$, the sets $\xi^{\perp}$ and $\xi^{*}$ will be considered as subsets of $X$. One can also think of $\xi^{*}$ as the set of vectors perpendicular to $\xi^{\perp}$, indeed for all $v \in \xi^{*}, w \in \xi^{\perp}$ we have $\|v-w\| \geq\langle\xi, v-w\rangle /\|\xi\|=\|v\|$.

Given $\xi \in X^{*} \backslash\{0\}$ and $v \in \xi^{*}$, it is natural to consider the decomposition $X=\xi^{\perp} \oplus \mathbb{R} v$. So given $f: \xi^{\perp} \rightarrow \mathbb{R} v$ we call graph of $f$ along $v$ the set $\Gamma_{f}:=\left\{z+t v: z \in \xi^{\perp}\right.$, tv $\left.=f(z)\right\}$ and subgraph of $f$ along $v$ the set $\Gamma_{f}^{-}:=\left\{z+t v: z \in \xi^{\perp}, t \leq s\right.$, where $\left.s v=f(z)\right\}$.

Given a function $f: \xi^{\perp} \rightarrow \mathbb{R} v\left(\xi \in X^{*}, v \in \xi^{*}\right)$ we say that $f$ is L-lipschitz if

$$
\|f(x)-f(y)\| \leq L\|x-y\| \quad \text { for all } x, y \in \xi^{\perp} .
$$

We say that the general Minkowski space $(X,\|\cdot\|)$ is euclidean whenever there exists an inner product $(\cdot, \cdot)$ on $X$ such that $\|x\|=\sqrt{(x, x)}$ for all $x \in X$. In this case $v^{*}$ and $\xi^{*}$ consist of a single element and $v \mapsto v^{*}$ turns out to be an isomorphism between $X$ and $X^{*}$ such that $\left\langle v^{*}, w\right\rangle=(v, w)$.

\subsection{Metric structure and Hausdorff measures}

We will consider on $(X,\|\cdot\|)$ the topology induced by the pre-base $\left\{B_{\rho}(x): x \in X, \rho>0\right\}$. It is not difficult to see that this topology does not depend on $\|\cdot\|$, so that it is the usual euclidean topology of $X$ (i.e. the topology induced by the usual topology of $\mathbb{R}^{n}$ through any linear isomorphism between $X$ and $\mathbb{R}^{n}$ ). 
In order to give a structure of metric space to $X$, it is useful to introduce the symmetrized norm

$$
\|x\|_{s}:=\max \{\|x\|,\|-x\|\},
$$

which induces on $X$ the distance $d_{s}(x, y)=\|x-y\|_{s}$. Note that

$$
\operatorname{diam}(E):=\sup _{x, y \in E} d_{s}(x, y)=\sup _{x, y \in E}\|x-y\| .
$$

By means of the distance $d_{s}$ we can define on $X$, for $k \in[0, n]$, the Hausdorff measures $\mathcal{H}^{k}$ and the spherical Hausdorff measures $\mathcal{S}^{k}$ (see [16]). Notice that these measures do depend on the general norm $\|\cdot\|$ of the space. Anyway, given two different general norms on $X$, we can find a constant $C>0$ such that $C^{-1} \mathcal{H}^{k} \leq \tilde{\mathcal{H}}^{k} \leq C \mathcal{H}^{k}$, where $\mathcal{H}^{k}$ and $\tilde{\mathcal{H}}^{k}$ are the two induced Hausdorff measures. This implies that $\mathcal{H}^{k}$-negligible sets do not depend on $\|\cdot\|$.

Since we have defined lipschitz functions and Hausdorff measures, we can introduce the notion of $k$-rectifiable sets of $X$ as the $\mathcal{H}^{k}$-measurable sets which are contained, up to a $\mathcal{H}^{k}$-negligible set, in a countable union of graphs of lipschitz functions from $\mathbb{R}^{k}$ to $X$. Notice that the family of rectifiable sets does not depend on the general norm of the space $X$ since the metrics $d_{s}$ induced by different general norms are equivalent.

We recall the main properties of rectifiable sets used in the sequel (here $\omega_{k}$ is the usual Lebesgue measure of the euclidean ball of $\mathbb{R}^{k}$ ).

Proposition 2.1. Let $E \subset X$ be $k$-rectifiable with $\mathcal{H}^{k}(E)<\infty$. Then, denoting by $B_{\rho}^{s}(x)$ the balls with respect to the symmetrized norm, we have

$$
\lim _{\rho \rightarrow 0} \frac{\mathcal{H}^{k}\left(B_{\rho}^{s}(x) \cap E\right)}{\omega_{k} \rho^{k}}=1 \quad \text { for } \mathcal{H}^{k} \text {-a.e. } x \in E
$$

and $\mathcal{H}^{k}(E)=\mathcal{S}^{k}(E)$.

Proof. The density property is proved in [21] in a more general metric setting. The agreement of $\mathcal{H}^{k}$ and $\mathcal{S}^{k}$ is a simple consequence of this fact, arguing as in 3.2.26 of [16].

\subsection{Perimeter}

On the Borel $\sigma$-algebra $\mathcal{B}(X)$ is defined a unique Haar measure $\mathcal{L}$ invariant under translations and such that $\mathcal{L}\left(B_{1}\right)=\omega_{n}$. We call this measure, Lebesgue measure on $X$ and we simply write $\int f(x) \mathrm{d} x$ instead of $\int f(x) \mathrm{d} \mathcal{L}(x)$ and $|A|$ instead of $\mathcal{L}(A)$. If $E_{k}, E \in \mathcal{B}(X)$ we write $E_{k} \rightarrow E$ as $k \rightarrow \infty$ and say that $E_{k}$ converge to $E$ locally in measure if $\left|\left(E_{k} \triangle E\right) \cap B_{\rho}\right| \rightarrow 0$ as $k \rightarrow \infty$ for all $\rho>0$. Again notice that, given two different general norms on $X$, it is possible to find a constant $C>0$ such that the estimate $C^{-1} \mathcal{L} \leq \tilde{\mathcal{L}} \leq C \mathcal{L}$ holds between the induced Lebesgue measures. So the local convergence in measure defined above does not depend on the general norm on $X$. If $\|\cdot\|$ is even we also notice that $\mathcal{L}=\mathcal{H}^{n}$; in fact $\mathcal{H}^{n}$ and $\mathcal{L}$ are both invariant Haar measures on $X$ and $\mathcal{H}^{n}\left(B_{1}^{s}\right)=\omega_{n}$.

With respect to the Lebesgue measure $\mathcal{L}$ we can consider the Lebesgue spaces $L^{p}(X):=L^{p}(X, \mathcal{L})$ (again, the topology of these spaces does not depend on the general norm on $X)$. If $V$ is a topological vector space and $\Omega \subseteq X$ is an open set, we can define, as usual, the space of differentiable functions $\mathcal{C}^{k}(\Omega ; V)$ and $\mathcal{C}_{c}^{k}(\Omega ; V)$ for $k=0, \ldots,+\infty$ (if $V$ is not specified we assume $V=\mathbb{R}$ ). Note that if $f \in \mathcal{C}^{k+1}(\Omega)$ the differential $D f$ belongs to $\mathcal{C}^{k}\left(\Omega ; X^{*}\right)$, in fact $D f(x)$ induces the element of $X^{*}$ defined by

$$
\langle D f(x), v\rangle:=\frac{\partial f}{\partial v}(x) \quad \forall v \in X .
$$

We also consider, as usual, the space of distributions $\mathcal{D}(\Omega ; V)$ as $\mathcal{L}\left(\mathcal{C}_{c}^{\infty}(\Omega), V\right)$ where $\mathcal{L}(W, V)$ is the space of all continuous linear applications $L: W \rightarrow V$. If $\Omega \subset X$ and $T \in \mathcal{D}(\Omega ; \mathbb{R})$ we define $D T \in \mathcal{D}\left(\Omega ; X^{*}\right)$ by $\langle\langle D T, \varphi\rangle, v\rangle:=-\langle T, \partial \varphi / \partial v\rangle\left(\right.$ where $\left.\varphi \in \mathcal{C}_{c}^{\infty}(\Omega), v \in X\right)$. 
If $E \subset X$ we denote by $\chi_{E}: X \rightarrow\{0,1\}$ the characteristic function of $E$. When $E \in \mathcal{B}(X)$ and the distributional derivative $D \chi_{E}$ is a Radon measure on $X$ (a vector measure with values in $X^{*}$ ) we say that $E$ is a set of locally finite perimeter and we define the perimeter of $E$ in any bounded Borel set $B$ as $P(E, B):=$ $\left\|-D \chi_{E}\right\|(B)$. Here $\|\mu\|$ represents the total variation of $\mu$, defined by

$$
\|\mu\|=\underset{v \in X \backslash\{0\}}{\mathcal{M}-\sup } \frac{\langle\mu, v\rangle}{\|v\|}
$$

where the supremum is taken in the family of all positive measures, with the partial ordering given by

$$
\mu \leq \nu \quad \Longleftrightarrow \mu(B) \leq \nu(B) \quad \forall B \in \mathcal{B}(X)
$$

For notational reasons, in the following we write $\bar{D} \chi_{E}$ in place of $-D \chi_{E}$.

Proposition 2.2. Let $A \subset X$ be an open set and let $E \subset X$ be a set with locally finite perimeter. Then

$$
P(E, A)=\sup \left\{\int_{E} \operatorname{div} \Phi(x) \mathrm{d} x: \Phi \in \mathcal{C}_{c}^{1}(A ; X),\|\Phi\|_{L^{\infty}} \leq 1\right\} .
$$

Moreover $E \mapsto P(E, A)$ is lower semicontinuous with respect to local convergence in measure.

Proof. Consider the linear functional $L_{E}: \mathcal{C}_{0}(A ; X) \rightarrow \mathbb{R}$ induced by the measure $\bar{D} \chi_{E}\llcorner A:$

$$
L_{E}(\Phi):=\int_{A} \Phi(x) \mathrm{d} \bar{D} \chi_{E}(x)
$$

By Riesz theorem, $L_{E}$ is continuous and $\left\|L_{E}\right\|=\left\|\bar{D} \chi_{E}\right\|(A)=P(E, A)$. Since $\mathcal{C}_{c}^{1}(A ; X)$ is dense in $\mathcal{C}_{0}(A ; X)$ and $L_{E}$ is continuous, we get

$$
\begin{aligned}
\left\|L_{E}\right\| & =\sup \left\{L_{E}(\Phi): \Phi \in \mathcal{C}_{0}(A ; X),\|\Phi\|_{L^{\infty}} \leq 1\right\} \\
& =\sup \left\{L_{E}(\Phi): \Phi \in \mathcal{C}_{c}^{1}(A ; X),\|\Phi\|_{L^{\infty}} \leq 1\right\}
\end{aligned}
$$

Since for $\Phi \in \mathcal{C}_{c}^{1}(A ; X)$ we have $L_{E}(\Phi)=\int_{E} \operatorname{div} \Phi(x) \mathrm{d} x,(3)$ is proved.

In (3) we have represented the perimeter as the supremum of a family (indexed by $\Phi$ ) of functionals continuous with respect to local convergence in measure; as a consequence the perimeter is lower semicontinuous.

Let $E$ be a set with locally finite perimeter, and let

$$
\nu_{E}(x):=\lim _{\rho \rightarrow 0^{+}} \frac{\bar{D} \chi_{E}\left(B_{\rho}(x)\right)}{\left\|\bar{D} \chi_{E}\right\|\left(B_{\rho}(x)\right)}
$$

(if the above limit does not exist at $x$, we set by convention $\nu_{E}(x)=0$ ). Then, by Besicovitch differentiation theorem [25] we know that $\bar{D} \chi_{E}=\nu_{E}\left\|\bar{D} \chi_{E}\right\|$ and $\nu_{E} \in L^{1}\left(X,\left\|\bar{D} \chi_{E}\right\| ; X^{*}\right)$ with $\left\|\nu_{E}(x)\right\|=1$ for $\left\|\bar{D} \chi_{E}\right\|$-a.e. $x \in X$.

Then, following [14] we define the reduced boundary to be the set $\partial^{*} E$ of all $x \in \operatorname{spt}\left\|\bar{D} \chi_{E}\right\|$ such that $\left\|\nu_{E}(x)\right\|=1$. When $x \in \partial^{*} E$ we call $\nu_{E}(x)$ the exterior unit normal vector to $E$ at $x$. We also introduce the density boundary $\partial^{m} E:=X \backslash\left(E^{0} \cup E^{1}\right)$ where $E^{\alpha}$ is defined by

$$
E^{\alpha}:=\left\{x \in X: \lim _{\rho \rightarrow 0} \frac{\left|E \cap B_{\rho}(x)\right|}{\omega_{n} \rho^{n}}=\alpha\right\} \quad \forall \alpha \in[0,1] .
$$

Notice that $\partial^{m} E$, i.e. the set of points where the density of $E$ is neither 0 nor 1 , does not depend on the general norm of the space. For $\xi \in X^{*} \backslash\{0\}$ define $H_{\xi}:=\{x \in X:\langle\xi, x\rangle \leq 0\}$, so that $\nu_{H_{\xi}}(x)=\xi /\|\xi\|$ for all $x \in \partial H_{\xi}=\xi^{\perp}$. 
On the other hand $\partial^{*} E$ depends on the general norm of the space. For example consider $X=\mathbb{R}^{2},\|(x, y)\|:=$ $\frac{2}{\sqrt{\pi}} \max \{|x|,|y|\}$ and $E:=\{(x, y): x \leq 0, y \leq 0\}$. Then one can find that $\bar{D} \chi_{E}\left(B_{\rho}\right)=\rho \frac{\sqrt{\pi}}{2}(1,1) \in X^{*}$ and $\left\|\bar{D} \chi_{E}\right\|\left(B_{\rho}\right)=\rho \frac{\pi}{2}$ (in fact for $(\xi, \eta) \in X^{*}$ we have $\|(\xi, \eta)\|=\frac{\sqrt{\pi}}{2}(|x|+|y|)$ ). So that $\nu_{E}(0)=\frac{1}{\sqrt{\pi}}(1,1)$ and $\left\|\nu_{E}(0)\right\|=1$ which means $0 \in \partial^{*} E$. If instead we consider the usual euclidean norm on $\mathbb{R}^{2}$ then we would find $\nu_{E}(0)=(1,1)$ but $\left|\nu_{E}(0)\right|=\sqrt{2}$ so that $0 \notin \partial^{*} E$.

In the euclidean case, the following fundamental results were proved by De Giorgi and Federer (see [14,15]).

Theorem 2.3 (De Giorgi-Federer). Let $X=\mathbb{R}^{n}$ endowed with the euclidean norm and let $E \subset X$ with locally finite perimeter. Then the reduced boundary $\partial^{*} E$ is $(n-1)$-rectifiable, is contained in $E^{1 / 2}$ and

$$
P(E, B)=\mathcal{H}^{n-1}\left(\partial^{*} E \cap B\right) \quad \forall B \in \mathcal{B}(X) .
$$

Moreover $\mathcal{H}^{n-1}\left(\partial^{m} E \backslash \partial^{*} E\right)=0$.

We will extend this result to general Minkowski spaces. To this aim, let us consider a linear isomorphism $\lambda: Y \rightarrow X$, where $X$ and $Y$ are general Minkowski spaces. We recall that the adjoint map $\lambda^{*}: X^{*} \rightarrow Y^{*}$ is defined by $\left\langle\lambda^{*} \xi, v\right\rangle=\langle\xi, \lambda v\rangle$ for all $\xi \in X^{*}$ and $v \in Y$. If $T$ is a distribution on $Y$ (with values in a vector space $V$ ) we recall that $\lambda_{\#} T$ is the distribution on $X$ (still with values in $V$ ) defined by $\left\langle\lambda_{\#} T, \Phi\right\rangle:=\langle T, \Phi \circ \lambda\rangle$ for all $\Phi \in \mathcal{C}_{c}^{\infty}(X, \mathbb{R})$. We will say that $\lambda$ preserves the Lebesgue measure if $|\lambda(B)|=|B|$ for one (and then for all, provided $|B|>0$ ) Borel set $B \subset Y$. This is equivalent to require that $\lambda_{\#} \mathcal{L}_{Y}=\mathcal{L}_{X}$, where $\mathcal{L}_{Y}$ and $\mathcal{L}_{X}$ are respectively the Lebesgue measures on $Y$ and $X$.

The following result can be proved arguing as in Lemma 3.5 of [20].

Lemma 2.4. Let $Y=\mathbb{R}^{n}$ endowed with the euclidean norm. Let $B \subset Y$ be a bounded convex open set containing the origin and let $F \subset Y$ be a set with locally finite perimeter. Suppose that there exist $\rho_{0}>0$ and $C>0$ such that

$$
\left.P(F, y+\rho B) \leq C\left|D \chi_{F}(y+\rho B)\right| \quad \forall \rho \in\right] 0, \rho_{0}[
$$

for some $y \in \operatorname{spt}\left\|D \chi_{F}\right\|$. Then $y \in \partial^{m} F$.

Theorem 2.5. Let $X, Y$ be general Minkowski spaces, and let $\lambda: Y \rightarrow X$ be a linear isomorphism which preserves the Lebesgue measure. Then, for all sets $E=\lambda(F) \subset X$ with locally finite perimeter,

$$
P(E, B)=\int_{B} \varphi\left(\nu_{E}(x)\right) \mathrm{d} \lambda_{\#}\left\|\bar{D} \chi_{F}\right\|(x) \quad \forall B \in \mathcal{B}(X)
$$

where $\varphi: X^{*} \rightarrow \mathbb{R}$ is the convex and positively 1-homogeneous function defined for $\xi \neq 0$ by $\varphi(\xi)=\|\xi\|^{2} /\left\|\lambda^{*} \xi\right\|$.

Moreover if $Y$ is euclidean we find that

$$
\lambda\left(\partial^{*} F\right) \subset \partial^{*} E \subset \partial^{m} E=\lambda\left(\partial^{m} F\right)
$$

As a consequence $\partial^{*} E$ is $(n-1)$-rectifiable and $\mathcal{H}^{n-1}\left(\partial^{m} E \backslash \partial^{*} E\right)=0$.

Proof. First of all notice that if $f \in L^{1}(Y, \mathbb{R})$ we have

$$
\left\langle\lambda_{\#} D f, w\right\rangle=\left\langle D\left(f \circ \lambda^{-1}\right), \lambda w\right\rangle=\left\langle\lambda^{*} D\left(f \circ \lambda^{-1}\right), w\right\rangle
$$

for all vectors $w \in Y$. In fact, given $\Phi \in \mathcal{C}_{c}^{\infty}(X, \mathbb{R})$ we have

$$
\begin{aligned}
\left\langle\lambda_{\#} \frac{\partial f}{\partial w}, \Phi\right\rangle & =-\int_{Y} f(y) \frac{\partial(\Phi \circ \lambda)}{\partial w}(y) \mathrm{d} y=-\int_{Y} f(y) \frac{\partial \Phi}{\partial(\lambda w)}(\lambda y) \mathrm{d} y \\
& =-\int_{X}\left(f \circ \lambda^{-1}\right)(x) \frac{\partial \Phi}{\partial(\lambda w)}(x) \mathrm{d} x=\left\langle\frac{\partial\left(f \circ \lambda^{-1}\right)}{\partial(\lambda w)}, \Phi\right\rangle .
\end{aligned}
$$


So, for $f=\chi_{F}$ we obtain

$$
\begin{aligned}
\left\|\bar{D} \chi_{E}\right\| & =\underset{v \in X \backslash\{0\}}{\mathcal{M}-\sup } \frac{\left\langle\bar{D} \chi_{E}, v\right\rangle}{\|v\|}=\underset{v \in X \backslash\{0\}}{\mathcal{M}-\sup } \frac{\left\langle-\lambda_{\#} D \chi_{F}, \lambda^{-1} v\right\rangle}{\|v\|} \\
& =\underset{w \in Y \backslash\{0\}}{\mathcal{M}-\sup _{w}} \frac{\left\langle\lambda_{\#} \bar{D} \chi_{F}, w\right\rangle}{\|\lambda w\|} .
\end{aligned}
$$

Notice that $\lambda_{\#}(\xi \mu)=\left(\xi \circ \lambda^{-1}\right)\left(\lambda_{\#} \mu\right)$ whenever $\mu$ is a positive measure; hence for $\xi=\nu_{F}$ and $\mu=\left\|\bar{D} \chi_{F}\right\|$ we obtain

$$
\begin{aligned}
\left\|\bar{D} \chi_{E}\right\| & =\underset{w \in Y \backslash\{0\}}{\mathcal{M}-\sup } \frac{\left\langle\left(\nu_{F} \circ \lambda^{-1}\right)\left(\lambda_{\#}\left\|\bar{D} \chi_{F}\right\|\right), w\right\rangle}{\|\lambda w\|} \\
& =\left(\sup _{w \in Y \backslash\{0\}} \frac{\left\langle\nu_{F} \circ \lambda^{-1}, w\right\rangle}{\|\lambda w\|}\right) \lambda_{\#}\left\|\bar{D} \chi_{F}\right\| .
\end{aligned}
$$

But since $\lambda^{*}\left(D \chi_{E}(B)\right)=\lambda_{\#} D \chi_{F}(B)$ for any Borel set $B$, we know that $\nu_{F} \circ \lambda^{-1}$ and $\lambda^{*} \nu_{E}$ have $\left\|\bar{D} \chi_{E}\right\|$-a.e. the same direction, so

$$
\nu_{F} \circ \lambda^{-1}=\frac{\lambda^{*} \nu_{E}}{\left\|\lambda^{*} \nu_{E}\right\|} \quad\left\|\bar{D} \chi_{E}\right\| \text {-a.e. in } X
$$

As a consequence

$$
\begin{aligned}
\left\|\bar{D} \chi_{E}\right\| & =\left(\sup _{w \in Y \backslash\{0\}} \frac{\left\langle\nu_{E}, \lambda w\right\rangle}{\|\lambda w\|}\right) \frac{\lambda_{\#}\left\|\bar{D} \chi_{F}\right\|}{\left\|\lambda^{*} \nu_{E}\right\|}=\frac{\left\|\nu_{E}\right\|}{\left\|\lambda^{*} \nu_{E}\right\|} \lambda_{\#}\left\|\bar{D} \chi_{F}\right\| \\
& =\frac{1}{\left\|\lambda^{*} \nu_{E}\right\|} \lambda_{\#}\left\|\bar{D} \chi_{F}\right\| .
\end{aligned}
$$

That is $\left\|\bar{D} \chi_{E}\right\|=\left(\varphi \circ \nu_{E}\right)\left(\lambda_{\#}\left\|\bar{D} \chi_{F}\right\|\right)$ and (4) is proved.

Now suppose that $\|\cdot\|_{Y}=|\cdot|_{Y}$ is euclidean, $y=\lambda^{-1}(x) \in \partial^{*} F, E_{\rho}:=(E-x) / \rho, F_{\rho}:=(F-y) / \rho$ and $H:=$ $\lambda\left(H_{\nu_{F}(y)}\right)$. By the proof of De Giorgi rectifiability theorem (see [20]) we know that $P\left(F_{\rho}, B\right) \rightarrow P\left(\lambda^{-1} H, B\right)$ for all balls $B$ and $\mathcal{H}^{n-1}\left(\partial^{m} F \backslash \partial^{*} F\right)=0$; we can thus apply Reshetnyak continuity theorem (see for instance [22]) and use (4) to obtain that $P\left(E_{\rho}, B\right) \rightarrow P(H, B)$ as $\rho \rightarrow 0^{+}$. So

$$
\frac{\bar{D} \chi_{E}\left(B_{\rho}(x)\right)}{\left\|\bar{D} \chi_{E}\right\|\left(B_{\rho}(x)\right)}=\frac{\bar{D} \chi_{E_{\rho}}\left(B_{1}\right)}{\left\|\bar{D} \chi_{E_{\rho}}\right\|\left(B_{1}\right)} \rightarrow \frac{\bar{D} \chi_{H}\left(B_{1}\right)}{\left\|\bar{D} \chi_{H}\right\|\left(B_{1}\right)}=\frac{\lambda \nu_{F}(y)}{\left\|\lambda \nu_{F}(y)\right\|}
$$

hence $x \in \partial^{*} E$.

Now we prove the inclusion $\partial^{*} E \subset \partial^{m} E$. Let $x=\lambda y \in \partial^{*} E$ and choose $C>0$ such that

$$
C^{-1}|| \xi\left\|\leq\left|\lambda^{*} \xi\right| \leq C|| \xi\right\| \quad \forall \xi \in X^{*} .
$$

We can find $\rho_{0}>0$ such that

$$
\left.P\left(E, B_{\rho}(x)\right) \leq 2\left\|\bar{D} \chi_{E}\left(B_{\rho}(x)\right)\right\| \quad \forall \rho \in\right] 0, \rho_{0}[
$$

and by (6) and (4) this implies

$$
\left.P(F, y+\rho B) \leq 2 C^{2}\left\|\bar{D} \chi_{F}(y+\rho B)\right\| \quad \forall \rho \in\right] 0, \rho_{0}[
$$


with $B=\lambda^{-1}\left(B_{1}\right)$. By Lemma 2.4 we obtain that $y \in \partial^{m} F$, hence $x \in \partial^{m} E$ (notice that the equality $\lambda\left(\partial^{m} E\right)=\partial^{m} F$ is trivial). This concludes the proof of (5).

Finally, since (by Th. 2.3) $\partial^{*} F$ is $(n-1)$-rectifiable and $\mathcal{H}^{n-1}\left(\partial^{m} F \backslash \partial^{*} F\right)=0$ we obtain that $\partial^{*} E$ is $(n-1)$-rectifiable and $\mathcal{H}^{n-1}\left(\partial^{m} E \backslash \partial^{*} E\right)=0$ as well.

By Theorem 2.5 and (7) we infer another representation of the perimeter in terms of the euclidean Hausdorff measure.

Corollary 2.6. Let $\lambda: \mathbb{R}^{n} \rightarrow X$ be a linear isomorphism preserving the Lebesgue measure. Then, given $B \in$ $\mathcal{B}(X)$ and a set $E=\lambda(F) \subset X$ with locally finite perimeter,

$$
P(E, B)=\int_{\lambda^{-1}(B) \cap \partial^{*} F}\left\|\left(\lambda^{-1}\right)^{*} \nu_{F}(y)\right\| \mathrm{d} \mathcal{H}^{n-1}(y) .
$$

In the following theorem we provide one more representation of the perimeter, in terms of the intrinsic Hausdorff measure (see also [9]).

Theorem 2.7. Let $E \subset X$ be a set with locally finite perimeter. Then

$$
P(E, B)=\int_{\partial^{*} E \cap B} \psi\left(\nu_{E}(x)\right) \mathrm{d} \mathcal{H}^{n-1}(x)
$$

where $\psi: X^{*} \rightarrow \mathbb{R}$ is the convex and positively 1-homogeneous function defined for $\xi \neq 0$ by

$$
\psi(\xi):=\|\xi\| \frac{P\left(H_{\xi}, B_{1}\right)}{\mathcal{H}^{n-1}\left(\xi^{\perp} \cap B_{1}\right)} .
$$

Proof. Notice that, by translation invariance, any bounded open set instead of $B_{1}$ could be considered in the definition of $\psi$. Consider a linear isomorphism $\lambda: \mathbb{R}^{n} \rightarrow X$ which preserves the Lebesgue measure and $F=\lambda^{-1}(E)$. Let $x \in \lambda\left(\partial^{*} F\right) \subset \partial^{*} E, B_{\rho}^{s}(x):=\left\{y \in X:\|y-x\|_{s}<\rho\right\}$ and $E_{\rho}:=(E-x) \rho$. We have

$$
\lim _{\rho \rightarrow 0^{+}} \frac{P\left(E, B_{\rho}^{s}(x)\right)}{\omega_{n-1} \rho^{n-1}}=\lim _{\rho \rightarrow 0} \frac{P\left(E_{\rho}, B_{1}^{s}\right)}{\omega_{n-1}}=\frac{P\left(H_{\nu_{E}(x)}, B_{1}^{s}\right)}{\omega_{n-1}}=\psi\left(\nu_{E}(x)\right)
$$

where the convergence of the limit is guaranteed by the Reshetnyak continuity theorem, as in the proof of Theorem 2.5. So, by the theory of spherical differentiation of measures (see [16], 2.10.19) we proved that $\left\|\bar{D} \chi_{E}\right\|\left\llcorner\lambda\left(\partial^{*} F\right)=\left(\psi \circ \nu_{E}\right) \mathcal{S}^{n-1}\left\llcorner\lambda\left(\partial^{*} F\right)\right.\right.$. By since $\partial^{*} E \backslash \lambda\left(\partial^{*} F\right)$ is $\mathcal{H}^{n-1}$-negligible we have $\left\|\bar{D} \chi_{E}\right\|\left\llcorner\lambda\left(\partial^{*} F\right)=\right.$ $\left\|\bar{D} \chi_{E}\right\|$ and the rectifiability of $\partial^{*} E$ together with Proposition 2.1 give

$$
\mathcal{S}^{n-1}\left\llcorner\lambda\left(\partial^{*} F\right)=\mathcal{S}^{n-1}\left\llcorner\partial^{*} E=\mathcal{H}^{n-1}\left\llcorner\partial^{*} E .\right.\right.\right.
$$

Lemma 2.8. Let $u(x):=\|x\|$. Then $\|D u\|=\mathcal{L}$.

Proof. Since $u$ is a lipschitz function, $u$ is differentiable for a.e. $x \in X$. Moreover, using the properties of the general norm, it is not difficult to show that

$$
\frac{\partial u}{\partial v}(x) \leq\|v\| \quad \forall v \in X, \quad \frac{\partial u}{\partial x}(x)=\|x\|
$$

for any differentiability point $x \in X \backslash\{0\}$. It follows that $\|\nabla u(x)\|=1$ wherever the differential $\nabla u(x)$ exists and $x \neq 0$. So $D u=\nabla u \mathcal{L}$ and $\|D u\|=\|\nabla u\| \mathcal{L}=\mathcal{L}$.

Now we prove that the natural normalization $\left|B_{1}\right|=\omega_{n}$ induces a natural normalization on $P\left(B_{1}, X\right)$. 
Theorem 2.9. There holds $P\left(B_{1}, X\right)=n \omega_{n}$.

Proof. We approximate $\chi_{B_{1}}$ by continuous functions. Let $\varphi_{\varepsilon}:[0, \infty[\rightarrow \mathbb{R}$ be the continuous function defined by

$$
\varphi(t):=\left\{\begin{array}{lll}
1 & \text { if } t \leq 1 \\
1+\frac{1-t}{\varepsilon} & \text { if } \quad 1<t \leq 1+\varepsilon \\
0 & \text { if } t>1+\varepsilon
\end{array}\right.
$$

and define $u_{\varepsilon}(x):=\varphi_{\varepsilon}(\|x\|)$. Since $u \mapsto\|\bar{D} u\|(X)$ is lower semicontinuous we get

$$
P\left(B_{1}, X\right) \leq \liminf _{\varepsilon \rightarrow 0^{+}}\left\|\bar{D} u_{\varepsilon}\right\|(X)=\liminf _{\varepsilon \rightarrow 0^{+}}\left\|\bar{D} u_{\varepsilon}\right\|\left(B_{1+\varepsilon} \backslash B_{1}\right) .
$$

As $u_{\varepsilon}(x)=(1+1 / \varepsilon-\|x\| / \varepsilon)$ in $B_{1+\varepsilon} \backslash B_{1}$, from Lemma 2.8 we get

$$
P\left(B_{1}, X\right) \leq \liminf _{\varepsilon \rightarrow 0^{+}} \frac{\mathcal{L}\left(B_{1+\varepsilon} \backslash B_{1}\right)}{\varepsilon}=\left.\frac{d}{d \rho} \mathcal{L}\left(B_{\rho}\right)\right|_{\rho=1}=n \omega_{n} .
$$

So we proved one inequality. Let now $u(x):=\|x\|$. If $\|x\| \leq 1$ we have

$$
u(x)-1=-\int_{0}^{1} \chi_{\{y: u(y)<t\}}(x) \mathrm{d} t
$$

whence, by linearity, we have

$$
D u=-\int_{0}^{1} D \chi_{\{y: u(y)<t\}} \mathrm{d} t \quad \text { in } B_{1} .
$$

Given any $v \in B_{1}$ and $\Phi \in \mathcal{C}_{c}^{\infty}\left(B_{1}\right)$ we get

$$
\left\langle\frac{\partial u}{\partial v}, \Phi\right\rangle=-\int_{0}^{1}\left\langle\frac{\partial}{\partial v} \chi_{\{y: u(y)<t\}}, \Phi\right\rangle \mathrm{d} t \leq \int_{0}^{1}\left\|\bar{D} \chi_{\{y: u(y)<t\}}\right\||\Phi| \mathrm{d} t .
$$

Since this is true for all $\Phi$ and $v$, we obtain

$$
\|D u\| \leq \int_{0}^{1}\left\|\bar{D} \chi_{\{y: u(y)<t\}}\right\| \mathrm{d} t \quad \text { in } B_{1} .
$$

Notice that $\left\|\bar{D} \chi_{\{y: u(y)<t\}}\right\|\left(B_{1}\right)=P\left(B_{t}, B_{1}\right)$ so that

$$
\omega_{n}=\|D u\|\left(B_{1}\right) \leq \int_{0}^{1} t^{n-1} P\left(B_{1}, X\right) \mathrm{d} t=\frac{P\left(B_{1}, X\right)}{n},
$$

and this concludes the proof.

We notice that, in general, $P\left(B_{1}, X\right) \neq P\left(-B_{1}, X\right)$.

Remark 2.10. Arguing as in Theorem 2.9 it is possible to show that

$$
P\left(E \cap B_{\rho}(x), \partial B_{\rho}(x)\right) \leq \frac{\mathrm{d}}{\mathrm{d} \rho}\left|E \cap B_{\rho}(x)\right|, \quad P\left(E \backslash B_{\rho}(x), \partial B_{\rho}(x)\right) \leq \frac{\mathrm{d}}{\mathrm{d} \rho}\left|E \cap B_{\rho}(x)\right|
$$

whenever $E$ has finite perimeter in $X$ and $t \mapsto\left|E \cap B_{t}(x)\right|$ is differentiable at $t=\rho$. 


\subsection{Isoperimetric inequalities}

Using Theorem 2.7, the following fundamental results can be recovered from $[28]$ (see also $[18,19]$, where the symmetry assumption is dropped).

Theorem 2.11 (global isoperimetric inequality). Let $E \subset X$ be such that $|E|<\infty$ and $P(E, X)=P\left(B_{1}, X\right)$. Then $|E| \leq\left|B_{1}\right|$ and equality holds if and only if $E=B_{1}+h$ for some $h \in \mathbb{R}^{n}$.

From Theorem 2.11 and Theorem 2.9 it easily follows that for a general set $E$ with $|E|<+\infty$ we have

$$
P(E, X) \geq n \omega_{n}\left(\frac{|E|}{\omega_{n}}\right)^{\frac{n-1}{n}} .
$$

Theorem 2.12 (local isoperimetric inequality). There exists a constant $\beta>0$, depending only on $(X,\|\cdot\|)$, such that

$$
\frac{P\left(E, B_{R}\right)}{R^{n-1}} \geq \beta \frac{\left|B_{R} \cap E\right|}{R^{n}} \cdot \frac{\left|B_{R} \backslash E\right|}{R^{n}} \quad \forall R>0, E \in \mathcal{B}(X) .
$$

\subsection{Non intrinsic notation}

Let $X$ be a general Minkowski space and let $\lambda: X \rightarrow \mathbb{R}^{n}$ be a linear isomorphism preserving the Lebesgue measure. Let also $(\cdot, \cdot)$ be the usual euclidean inner product of $\mathbb{R}^{n}$. Then the adjoint map $\lambda^{*}: \mathbb{R}^{n} \rightarrow X^{*}$ verifies $\left\langle\lambda^{*} \xi, \lambda^{-1} v\right\rangle=(\xi, v)$. So we may define the functions $\varphi: \mathbb{R}^{n} \rightarrow \mathbb{R}, \varphi(v):=\left\|\lambda^{-1} v\right\|, \varphi^{o}: \mathbb{R}^{n} \rightarrow \mathbb{R}$, $\varphi^{o}(\xi)=\left\|\lambda^{*} \xi\right\|, T: \mathbb{R}^{n} \rightarrow \mathcal{P}\left(\mathbb{R}^{n}\right), T(v):=\lambda^{*-1}\left(\left(\lambda^{-1} v\right)^{*}\right), T^{o}: \mathbb{R}^{n} \rightarrow \mathcal{P}\left(\mathbb{R}^{n}\right), T^{o}(\xi):=\lambda\left(\left(\lambda^{*} \xi\right)^{*}\right), W_{\varphi}:=\lambda\left(B_{1}\right)$, $F_{\varphi}:=\left(\lambda^{*}\right)^{-1}\left(B_{1}^{*}\right), P_{\varphi}(E, B):=P(\lambda(E), \lambda(B))$. This provides an equivalent definition of the space $(X,\|\cdot\|)$ as $\mathbb{R}^{n}$ endowed with the general norm $\varphi$, which is the usual approach followed in the literature [9,26].

By Corollary 2.6, we have

$$
P(E, B)=\int_{\lambda(\partial E \cap B)} \varphi^{o}\left(\nu_{\lambda(E)}(x)\right) \mathrm{d} \mathcal{H}^{n-1}(x)
$$

where $\nu_{\lambda(E)}(x)$ is the usual euclidean normal vector to $\lambda(E)$ in $x$.

\section{3. $\omega$-MINIMAL SETS}

In order to have boundary, closure and interior operators invariant under changes in negligible sets, we define

$$
\begin{array}{rll}
\partial E & :=\{x \in X: \forall \rho>0 & \left.\left|E \cap B_{\rho}(x)\right| \notin\left\{0,\left|B_{\rho}(x)\right|\right\}\right\}, \\
\bar{E}:=\{x \in X: \forall \rho>0 & \left.\left|E \cap B_{\rho}(x)\right| \neq 0\right\}, \\
E & :=\{x \in X: \exists \rho>0 & \left.\left|E \cap B_{\rho}(x)\right|=\left|B_{\rho}(x)\right|\right\} .
\end{array}
$$

Notice that if $|E \triangle F|=0$ then $\partial E=\partial F, \bar{E}=\bar{F}$ and $\stackrel{\circ}{E}=\stackrel{\circ}{F}$. Moreover $\partial E, \bar{E}$ are closed sets, $\stackrel{\circ}{E}$ is open and the usual relations $\partial E=\bar{E} \backslash \stackrel{\circ}{E}$ and $\stackrel{\circ}{E}=X \backslash \overline{X \backslash E}$ hold. Notice that in general $|E \backslash \stackrel{\circ}{E}|,|\bar{E} \backslash E|>0$. If $\Omega \subset X$ we write $E \Subset \Omega$ when $\bar{E} \subset \Omega$ and $\bar{E}$ is compact.

Let $\omega:(0, \infty) \rightarrow(0, \infty]$ be a nondecreasing function with $\lim _{\rho \rightarrow 0^{+}} \omega(\rho)=0$.

Definition 3.1. Let $\Omega \subset X$ be an open set. We say that $E \subset X$ is an $\omega$-minimal set in $\Omega$ if for all $B_{\rho}(x) \Subset \Omega$ with $x \in \partial E$ and all $F \subset X$ with $E \triangle F \Subset B_{\rho}(x)$ we have

$$
P\left(E, B_{\rho}(x)\right) \leq(1+\omega(\rho)) P\left(F, B_{\rho}(x)\right) .
$$

We denote by $\mathcal{M}_{\omega}(\Omega)$ the family of all $\omega$-minimal sets in $\Omega$ and we let $\mathcal{M}_{\omega}:=\mathcal{M}_{\omega}(X)$. 
Proposition 3.2 (density upper bound). There exists a constant $\Theta>0$ (depending only on $n, X, \omega)$ such that if $E \in \mathcal{M}_{\omega}(\Omega)$ and $B_{\rho}(x) \Subset \Omega$ with $\omega(\rho) \leq 1$ then

$$
P\left(E, B_{\rho}(x)\right) \leq \Theta \rho^{n-1}
$$

Proof. Suppose for simplicity $x=0$. Take $\eta<\rho$ and let $F_{\eta}=E \cup B_{\eta}$. Then we have

$$
P\left(F_{\eta}, B_{\rho}\right)=P\left(E, B_{\rho} \backslash \overline{B_{\eta}}\right)+P\left(F_{\eta}, \partial B_{\eta}\right) \leq P\left(E, B_{\rho} \backslash \overline{B_{\eta}}\right)+n \omega_{n} \eta^{n-1}
$$

since $P\left(F_{\eta}, \partial B_{\eta}\right) \leq P\left(B_{\eta}, X\right)=n \omega_{n} \eta^{n-1}$. Then, since $F_{\eta} \triangle E \Subset B_{\rho}$ we get

$$
P\left(E, B_{\rho}\right) \leq(1+\omega(\rho))\left(P\left(E, B_{\rho} \backslash \overline{B_{\eta}}\right)+n \omega_{n} \eta^{n-1}\right)
$$

and letting $\eta \rightarrow \rho^{-}$we conclude

$$
P\left(E, B_{\rho}\right) \leq n \omega_{n}(1+\omega(\rho)) \rho^{n-1}
$$

Using Theorem 2.7 and reasoning as in [3,3.2], one can get the following compactness result (see also [5]).

Proposition 3.3 (compactness of $\omega$-minimal sets). Let $\left(E_{k}\right)$ be a sequence of $\omega_{k}$-minimal sets in $\Omega_{k} \subset X$ and suppose that $\omega_{k} \rightarrow \omega$ pointwise and $\Omega_{k} \uparrow X$. Then there exists a subsequence $\left(E_{k_{j}}\right)$ converging in $L_{\text {loc }}^{1}(X)$ to $E \in \mathcal{M}_{\omega}$. Moreover, if $E_{k}$ converge in $L_{\text {loc }}^{1}(X)$ to $E$ then

$$
D \chi_{E_{k}} \stackrel{*}{\rightarrow} D \chi_{E} \quad \text { and } \quad\left\|\bar{D} \chi_{E_{k}}\right\| \stackrel{*}{\rightarrow}\left\|\bar{D} \chi_{E}\right\| .
$$

Proposition 3.4 (volume bounds). Let $E \in \mathcal{M}_{\omega}(\Omega)$. Then there exists a constant $\left.\gamma \in\right] 0,1[$, depending only on $X$ and $\omega$, such that

$$
\gamma \leq \frac{\left|E \cap B_{\rho}(x)\right|}{\omega_{n} \rho^{n}} \leq 1-\gamma
$$

whenever, $B_{\rho}(x) \Subset \Omega, \omega\left(\rho_{+}\right)<1$ and $x \in \partial E$.

Proof. Suppose for simplicity $x=0$ and consider the nondecreasing function $g(\rho):=\left|E \cap B_{\rho}\right|$. Theorem 2.11 gives

$$
\frac{1}{C_{1}} g(\rho)^{\frac{n-1}{n}} \leq P\left(E \cap B_{\rho}, X\right)=P\left(E, B_{\rho}\right)+P\left(E \cap B_{\rho}, \partial B_{\rho}\right)
$$

for some dimensional constant $C_{1}>0$. By Remark 2.10 we find that

$$
P\left(E \cap B_{\rho}, \partial B_{\rho}\right), P\left(E \backslash B_{\rho}, \partial B_{\rho}\right) \leq g^{\prime}(\rho)
$$

for all $\rho>0$ for which $g^{\prime}(\rho)$ exists. Comparing $E$ with $E \cap B_{\rho}$ we have

$$
P\left(E, B_{\eta}\right) \leq(1+\omega(\eta)) P\left(E \backslash B_{\rho}, B_{\eta}\right) \quad \forall \eta>\rho
$$

and letting $\eta \rightarrow \rho^{+}$, if $\omega\left(\rho_{+}\right)<1$ we get

$$
P\left(E, \overline{B_{\rho}}\right) \leq\left(1+\omega\left(\rho_{+}\right)\right) P\left(E \backslash B_{\rho}, \overline{B_{\rho}}\right)=\left(1+\omega\left(\rho_{+}\right)\right) P\left(E \backslash B_{\rho}, \partial B_{\rho}\right) \leq 2 g^{\prime}(\rho) .
$$

So, we get $g(\rho)^{\frac{n-1}{n}} \leq 3 C_{1} g^{\prime}(\rho)$, that is $\left(g(\rho)^{\frac{1}{n}}\right)^{\prime} \geq 1 /\left(3 C_{1} n\right)$, hence the first inequality follows by integration. 
To prove the second inequality, consider $X \backslash E$ instead of $E$ and repeat the proof; even if, in general, we cannot assume that $X \backslash E$ is $\omega$-minimal, the inequality

$$
P\left(X \backslash E, B_{\rho}\right) \leq C P\left(E, B_{\rho}\right)
$$

is sufficient to complete the proof.

Notice that the previous estimate ensures that if $x_{k} \in \partial E_{k}, E_{k} \rightarrow E$ in $L_{\text {loc }}^{1}(X), x_{k} \rightarrow x$ and $E_{k} \in \mathcal{M}_{\omega}$ then $x \in \partial E$. This will be often used in the following:

Proposition 3.5 (density lower bound). There exist $\theta>0$ and $\rho_{\omega}>0$ such that, if $E \in \mathcal{M}_{\omega}(\Omega)$ and $B_{\rho}(x) \Subset$ $\Omega$ with $\rho<\rho_{\omega}$ and $x \in \partial E$, then

$$
P\left(E, B_{\rho}(x)\right) \geq \theta \rho^{n-1}
$$

Proof. In the case $E \in \mathcal{M}_{0}(\Omega)$ we will reason as in [3, 3.4], assuming for simplicity $n>2$ (in dimension $n=2$ the proof is quite simpler). Let $\gamma:=\left((n-1)^{n-1} \omega_{n-1}\right)^{\frac{1}{n-2}}$ and consider a bijection $\lambda: X \rightarrow \mathbb{R}^{n}$ as in Section 2.5 . As a consequence of the isoperimetric inequalities for subsets of $\mathbb{R}^{n}$ of arbitrary codimension (see [1]), it follows that for a.e. $\eta>0$ there exists a set $\lambda\left(E_{\eta}\right) \subset \mathbb{R}^{n}$ with locally finite perimeter, such that $\lambda(E) \triangle \lambda\left(E_{\eta}\right) \subseteq B_{\eta}(\lambda x)$ and

$$
P\left(\lambda\left(E_{\eta}\right), \overline{B_{\eta}(\lambda x)}\right) \leq \gamma\left(m^{\prime}(\eta)\right)^{\frac{n-1}{n-2}}, \quad \text { where } \quad m(\eta):=P\left(\lambda(E), \overline{B_{\eta}(\lambda x)}\right) .
$$

Hence, setting $c:=\max \left\{\sup _{|e|=1} \varphi^{o}(e),\left(\inf _{|e|=1} \varphi^{o}(e)\right)^{-1}\right\}$, by the minimality of $E$ we get

$$
\begin{aligned}
c^{-1} m(\eta) & \leq P\left(E, \lambda^{-1}\left(B_{\eta}(\lambda x)\right)\right) \leq P\left(E_{\eta}, \overline{\lambda^{-1}\left(B_{\eta}(\lambda x)\right)}\right) \\
& \leq c P\left(\lambda\left(E_{\eta}\right), \overline{B_{\eta}(x)}\right) \leq c \gamma\left(m^{\prime}(\eta)\right)^{\frac{n-1}{n-2}}
\end{aligned}
$$

for a.e. $\eta>0$. This implies

$$
\frac{m(\eta)}{\eta^{n-1}} \geq \frac{1}{(n-1)^{n-1}}\left(\frac{1}{2 \gamma c^{2}}\right)^{\frac{(n-1)^{2}}{n-2}}
$$

and letting $\eta=c^{-1} \rho$ we get $\lambda\left(B_{\rho}(x)\right) \supseteq B_{\eta}(\lambda x)$ so that

$$
\frac{P\left(E, B_{\rho}(x)\right)}{\rho^{n-1}} \geq \frac{P\left(\lambda(E), B_{\eta}(\lambda x)\right)}{c^{n} \eta^{n-1}} \geq \frac{1}{c^{n}(n-1)^{n-1}}\left(\frac{1}{2 \gamma c^{2}}\right)^{\frac{(n-1)^{2}}{n-2}}=: 2 \theta .
$$

In the general case $E \in \mathcal{M}_{\omega}(\Omega)$, we argue as in [8]. Suppose, by contradiction, that there exist sets $E_{k} \in \mathcal{M}_{\omega}(\Omega)$, points $x_{k} \in \partial E_{k}$ and radii $\rho_{k} \rightarrow 0$ such that

$$
\rho_{k}^{1-n} P\left(E_{k}, B_{\rho_{k}}\left(x_{k}\right)\right)<\theta .
$$

By the rescaling properties of quasi-minimizers the sets $F_{k}:=\left(E_{k}-x_{k}\right) / \rho_{k}$ belong to $\mathcal{M}_{\omega_{k}}\left(\left(\Omega-x_{k}\right) / \rho_{k}\right)$, where $\omega_{k}(t):=\omega\left(\rho_{k} t\right)$. We note that $\omega_{k} \rightarrow 0$ so that by Proposition 3.3, up to a subsequence, we may suppose that $F_{k} \rightarrow F$ with $F \in \mathcal{M}_{0}$. From the lower semi-continuity of perimeter we obtain

$$
\theta \geq \liminf _{k \rightarrow \infty} \rho_{k}^{1-n} P\left(E_{k}, B_{\rho_{k}}\left(x_{k}\right)\right)=\liminf _{k \rightarrow \infty} P\left(F_{k}, B_{1}\right) \geq P\left(F, B_{1}\right) .
$$

But since by Proposition $3.40 \in \partial F$ and $F \in \mathcal{M}_{0}$ we have already proved that $P\left(F, B_{1}\right) \geq 2 \theta$, so that we have a contradiction. 
Corollary 3.6. For any $E \in \mathcal{M}_{\omega}(\Omega)$, there holds

$$
\mathcal{H}^{n-1}\left((\partial E \cap \Omega) \backslash \partial^{*} E\right)=0
$$

Proof. By (8) the measure $\left\|\bar{D} \chi_{E}\right\|$ has strictly positive (spherical) $(n-1)$-dimensional upper density greater than $\theta / \omega_{n-1}$ at any $x \in \partial E$, hence

$$
\left\|\bar{D} \chi_{E}\right\|(B) \geq \frac{\theta}{\omega_{n-1}} \mathcal{H}^{n-1}(B) \quad \forall B \in \mathcal{B}(X), B \subset \partial E .
$$

Choosing $B=\partial E \backslash \partial^{*} E$ and taking into account that $\left\|\bar{D} \chi_{E}\right\|$ is concentrated on $\partial^{*} E$ the conclusion follows.

By Corollary 3.6, when we deal with $\omega$-minimal sets, we can equivalently integrate with respect to $\mathcal{H}^{n-1}$ (or $\left.\left\|\bar{D} \chi_{E}\right\|\right)$ either on $\partial E$ or on $\partial^{*} E$.

Remark 3.7 (additive quasi minimizers). Let us define an additive $\omega$-minimal set in $\Omega$ as a set $E \in \mathcal{B}(X)$ such that

$$
P\left(E, B_{\rho}(x)\right) \leq P\left(F, B_{\rho}(x)\right)+\rho^{n-1} \omega(\rho)
$$

for any ball $B_{\rho}(x) \Subset \Omega$ and any $F \subset X$ with $E \triangle F \Subset B_{\rho}(x)$. If $E$ satisfies also (8) for some $\theta>0$, it is easy to check that $E$ is also a $\omega /(\theta-\omega)$-minimizer in the sense of Definition 3.1.

An example of sets which satisfy the additive $\omega$-minimal condition and the density lower bound (8) is given by sets with prescribed mean curvature in $L^{n}$, that is, the minima of the functional

$$
E \mapsto P(E, \Omega)+\int_{E \cap \Omega} H(x) \mathrm{d} x
$$

with $H \in L^{n}(\Omega)($ see $[8])$.

Remark 3.8. Let $\lambda: X \rightarrow \mathbb{R}^{n}$ be as in Corollary 2.6, let $E \in \mathcal{M}_{\omega}(\Omega)$ with $\partial E \cap \Omega \neq \emptyset$ and set $E^{\prime}:=\lambda(E) \subset \mathbb{R}^{n}$, $\Omega^{\prime}:=\lambda(\Omega)$. Then, for any $\delta>0$ there exists a constant $k>0$, depending only on $\omega$ and $\delta$, such that $E^{\prime}$ is a $\left(\Omega^{\prime}, k, \delta\right)$-minimizer in the sense of [13]. More precisely, $E^{\prime}$ satisfies the following properties:

1. $\partial E^{\prime} \cap \Omega^{\prime} \neq \emptyset$

2. $P\left(E^{\prime}, B\right)<+\infty$ for each $B \Subset \Omega^{\prime}$;

3. $P\left(E^{\prime}, \Omega^{\prime}\right) \leq k P\left(\varphi\left(E^{\prime}\right), \Omega^{\prime}\right)$ whenever $\varphi \in \operatorname{Lip}\left(\mathbb{R}^{n} ; \mathbb{R}^{n}\right), \operatorname{diam}(W \cup \varphi(W))<\delta$ and $W \cup \varphi(W) \Subset \Omega^{\prime} \Subset \mathbb{R}^{n}$, where $W:=\left\{x \in \mathbb{R}^{n}: \varphi(x) \neq x\right\}$.

By the previous remark and [13] (Th. 2.11) it follows that $\partial E^{\prime}$ fulfils some mild regularity properties. Precisely, for any $x \in \partial E^{\prime}$ and $\left.R \in\right] 0, \delta\left[\right.$ such that $B_{3 R}(x) \subset \Omega^{\prime}$, there exists a constant $C>0$, depending only on $\omega$ and $\delta$, such that for any $y \in \partial E^{\prime} \cap B_{R}(x)$ and any ball $B_{r}(y) \subset B_{R}(x)$ there exists a $C$-lipschitz graph $\Gamma$ such that

$$
\mathcal{H}^{n-1}\left(\partial E^{\prime} \cap \Gamma \cap B_{r}(y)\right) \leq \frac{1}{C} r^{n-1}
$$

Being the statement bi-lipschitz invariant, the same holds for $\partial E$, by composing with $\lambda^{-1}$.

Lemma 3.9 (cut and paste). Let $\Omega \subset X$ be an open set and let $E, H \subset X$ be sets with locally finite perimeter. Suppose moreover that $\bar{E} \cap \partial \Omega=\bar{H} \cap \partial \Omega$, and $\mathcal{H}^{n-1}(\partial E \cap \partial \Omega)=\mathcal{H}^{n-1}(\partial H \cap \partial \Omega)=0$. Then, letting $F:=$ $(E \backslash \Omega) \cup(H \cap \Omega)$ we have

$$
P(F, B)=P(E, B \backslash \Omega)+P(H, B \cap \Omega)
$$

for all Borel subsets $B \subset X$. 
In particular, if $E \in \mathcal{M}_{\omega}$ and $\Omega \Subset B_{\rho}\left(x_{0}\right)$ with $x_{0} \in \partial E$, we have

$$
P(E, \Omega) \leq P(H, \Omega)+\omega\left(\rho_{+}\right) P\left(F, \overline{B_{\rho}\left(x_{0}\right)}\right)=\left(1+\omega\left(\rho_{+}\right)\right) P(H, \Omega)+\omega\left(\rho_{+}\right) P\left(E, \overline{B_{\rho}\left(x_{0}\right)} \backslash \Omega\right) .
$$

Proof. First we prove that $\partial F \cap \partial \Omega \subset \partial E \cup \partial H$. Let $x \in \partial \Omega \backslash(\partial E \cup \partial H)$ and suppose $x \in \stackrel{\circ}{E}$ (otherwise we may consider the complementary sets of $E$ and $H$ ). By hypothesis $x \in \stackrel{\circ}{H}$ so we get, for some $\rho>0$, $\left|B_{\rho}(x) \cap E\right|=\left|B_{\rho}(x) \cap H\right|=\omega_{n} \rho^{n}$. Then obviously $\left|B_{\rho}(x) \cap F\right|=\omega_{n} \rho^{n}$, which means $x \notin \partial F$. So we have proved that $\partial F \cap \partial \Omega \subset \partial E \cup \partial H$ and in particular $P(F, \partial \Omega)=0$ since $\mathcal{H}^{n-1}(\partial F \cap \partial \Omega) \leq \mathcal{H}^{n-1}((\partial E \cup \partial H) \cap \partial \Omega)=0$.

So, by the locality of perimeter, for all Borel sets $B \subset X$ we have

$$
\begin{aligned}
P(F, B) & =P(F, B \cap \Omega)+P(F, B \backslash \bar{\Omega})+P(F, B \cap \partial \Omega) \\
& =P(H, B \cap \Omega)+P(E, B \backslash \bar{\Omega}) .
\end{aligned}
$$

Now let us prove the second statement. Suppose for simplicity $x_{0}=0$. Choose a radius $\eta>\rho$ such that $P\left(E, \partial B_{\eta}\right)=0$. Since $E \triangle F \subset \Omega \Subset B_{\eta}$ from the minimality of $E$ we get $P\left(E, B_{\eta}\right) \leq(1+\omega(\eta)) P\left(F, B_{\eta}\right)$. But since we have $P\left(E, B_{\eta}\right)=P(E, \Omega)+P\left(E, B_{\eta} \backslash \Omega\right)$ and $P\left(F, B_{\eta}\right)=P(H, \Omega)+P\left(E, B_{\eta} \backslash \Omega\right)$, the conclusion follows letting $\eta \rightarrow \rho^{+}$.

Proposition 3.10 (Gauss-Green). Let $B$ be an open set and $E, F$ sets of $X$ with locally finite perimeter in $X$. If $E \triangle F \Subset B$ then $D \chi_{E}(B)=D \chi_{F}(B)$. The same result holds if we know that $\bar{E} \cap \partial B=\bar{F} \cap \partial B$ and $\mathcal{H}^{n-1}(\partial F \cap \partial B)=\mathcal{H}^{n-1}(\partial E \cap \partial B)=0$.

Proof. Given $\varphi_{k} \in C_{c}^{\infty}(B)$ such that $\varphi_{k}=1$ on $E \triangle F$ and $\varphi_{k} \rightarrow \chi_{B}$ in $L^{1}$ we have

$$
\left|\left\langle D \chi_{E}-D \chi_{F}, \varphi_{k}\right\rangle\right|=\left|\int_{E} D \varphi_{k}-\int_{F} D \varphi_{k}\right| \leq \int_{E \triangle F}\left|D \varphi_{k}\right|=0
$$

and the first result follows.

For the second statement, we set $F^{\prime}:=(F \cap B) \cup(E \backslash B)$. We notice that $F^{\prime} \triangle E \subset B$. For any $\rho>0$ consider the $\rho$-neighbourhood of $B: B_{\rho}:=\left\{x \in X: \inf _{y \in B}\|x-y\| \leq \rho\right\}$. Since $F^{\prime} \triangle E \Subset B_{\rho}$ we know that $D \chi_{E}\left(B_{\rho}\right)=D \chi_{F}^{\prime}\left(B_{\rho}\right)$ and letting $\rho \rightarrow 0^{+}$we obtain $D \chi_{E}(\bar{B})=D \chi_{F^{\prime}}(\bar{B})$. Since $P(E, \partial B)=0$ and $F^{\prime} \cap B=F \cap B$ we conclude $D \chi_{E}(B)=D \chi_{F^{\prime}}(B)=D \chi_{F}(B)$.

\section{LiPSCHITZ APPROXIMATION OF SETS With SMALL EXCESS}

In this section we introduce the notion of excess, that is a quantity which measures the "distance" of the set from being flat in a given ball. In Proposition 4.6 we will prove that the boundary of an $\omega$-minimal set coincides with the graph of a lipschitz function up to a set whose $(n-1)$-Hausdorff measure is controlled by the excess.

In order to study the regularity of $\omega$-minimal sets we consider the following quantities (see [5])

$$
\begin{aligned}
& \operatorname{Ecc}_{E}^{v}(x, \rho):=\rho^{1-n}\left(\left\|\bar{D} \chi_{E}\right\|\left(B_{\rho}(x)\right)-\left\langle\bar{D} \chi_{E}\left(B_{\rho}(x)\right), v\right\rangle\right), \\
& \operatorname{Ecc}_{E}(x, \rho):=\inf _{v \in X,\|v\|=1} \mathbf{E c c}_{E}^{v}(x, \rho)=\rho^{1-n}\left(\left\|\bar{D} \chi_{E}\right\|\left(B_{\rho}(x)\right)-\left\|\bar{D} \chi_{E}\left(B_{\rho}(x)\right)\right\|\right) \text {. }
\end{aligned}
$$

We also define the singular set $\Sigma(E)$ as

$$
\Sigma(E):=\left\{x \in \partial E: \limsup _{\rho \rightarrow 0^{+}} \mathbf{E c c}_{E}(x, \rho)>0\right\} .
$$


Notice that $\Sigma(E) \cap \partial^{*} E=\emptyset$; indeed if $x \in \partial^{*} E$ we have, by definition,

$$
\lim _{\rho \rightarrow 0^{+}} \frac{\bar{D} \chi_{E}\left(B_{\rho}(x)\right)}{\left\|\bar{D} \chi_{E}\right\|\left(B_{\rho}(x)\right)}=\nu_{E}(x)
$$

where $\left\|\nu_{E}(x)\right\|=1 ;$ i.e.

$$
\lim _{\rho \rightarrow 0^{+}} \frac{\left\|\bar{D} \chi_{E}\right\|\left(B_{\rho}(x)\right)-\left\|\bar{D} \chi_{E}\left(B_{\rho}(x)\right)\right\|}{\left\|\bar{D} \chi_{E}\right\|\left(B_{\rho}(x)\right)}=0 .
$$

Since by (5) $x \in \partial^{m} E$, the relative isoperimetric inequality implies that $\left\|\bar{D} \chi_{E}\right\|\left(B_{\rho}(x)\right) \geq c \rho^{n-1}$ for a suitable $c>0$ and $\rho$ sufficiently small. We conclude that $\lim _{\rho \rightarrow 0^{+}} \operatorname{Eccc}_{E}(x, \rho)=0$. Since $\partial^{*} E$ and $\Sigma(E)$ are disjoint it follows that $\left\|\bar{D} \chi_{E}\right\|(\Sigma(E))=0$ and Theorem 2.7 gives

$$
\mathcal{H}^{n-1}(\Sigma(E))=0
$$

These quantities are meant to measure the "flatness" of the set $E$ in the ball $B_{\rho}(x)$. In fact, when $B_{1}^{*}$ is strictly convex and $\mathbf{E c c}_{E}(x, \rho)=0$ then $E \cap B_{\rho}(x)$ is an half-plane. If $B_{1}^{*}$ is not strictly convex this is not always true, but we can state the following result:

Lemma 4.1. Let $x_{0} \in X, v \in \partial B_{1}$ and let $T \subset X$ be a hyper-space perpendicular to $v$. If $\mathbf{E c c}_{E}^{v}\left(x_{0}, \rho\right)=0$ then $E$ coincides in $B_{\rho}\left(x_{0}\right)$ with the subgraph of a $L_{0}$-lipschitz function $f: T \rightarrow \mathbb{R} v$. The constant $L_{0}$ depends only on $(X,\|\cdot\|)$.

Proof. Let us choose mollifiers $\varphi_{\varepsilon} \in \mathcal{C}_{0}^{\infty}(X)$ such that $\operatorname{spt} \varphi_{\varepsilon} \subset B_{\varepsilon}, \varphi_{\varepsilon} \geq 0, \int_{B_{\varepsilon}} \varphi_{\varepsilon}=1$ and $\varphi_{\varepsilon}(x)=$ $3\left(4 \omega_{n}(\varepsilon / 2)^{n}\right)^{-1}$ for $x \in B_{\varepsilon / 2}$. We consider the mollified functions $u_{\varepsilon}:=\chi_{E} * \varphi_{\varepsilon}$.

Let $x \in X, \varepsilon>0$ be such that $u_{\varepsilon}(x)=1 / 2$ and $B_{\varepsilon}(x) \subseteq B_{\rho}\left(x_{0}\right)$. Then

$$
\begin{aligned}
\frac{3}{4} \frac{\left|E \cap B_{\varepsilon / 2}(x)\right|}{\left|B_{\varepsilon / 2}\right|} & =\int_{E \cap B_{\varepsilon / 2}(x)} \varphi_{\varepsilon}(y-x) \mathrm{d} y \\
& =\int_{E} \varphi_{\varepsilon}(y-x) \mathrm{d} y-\int_{E \cap\left(B_{\varepsilon}(x) \backslash B_{\varepsilon / 2}(x)\right)} \varphi_{\varepsilon}(y-x) \mathrm{d} y \\
& \geq \frac{1}{2}-\left(1-\frac{3}{4}\right)=\frac{1}{4}
\end{aligned}
$$

that is $\left|E \cap B_{\varepsilon / 2}(x)\right| \geq \frac{1}{3}\left|B_{\varepsilon / 2}(x)\right|$; reasoning the same way with $X \backslash E$ we also find $\left|B_{\varepsilon / 2}(x) \backslash E\right| \geq \frac{1}{3}\left|B_{\varepsilon / 2}(x)\right|$. Hence, by Theorem 2.12 we get $P\left(E, B_{\varepsilon / 2}(x)\right)>\alpha(\varepsilon / 2)^{n-1}$, with $\alpha>0$ depending only on $(X,\|\cdot\|)$.

Consider now the positive measures $\mu_{\varepsilon}:=\varphi_{\varepsilon}(\cdot-x)\left\|\bar{D} \chi_{E}\right\|$. Letting $\alpha_{\varepsilon}:=\mu_{\varepsilon}\left(\mathbb{R}^{n}\right)$, for $\varepsilon$ sufficiently small we have

$$
\alpha_{\varepsilon} \geq \mu_{\varepsilon}\left(B_{\frac{\varepsilon}{2}}(x)\right)=\frac{3}{4 \omega_{n}(\varepsilon / 2)^{n}} P\left(E, B_{\varepsilon / 2}(x)\right) \geq \frac{3 \alpha}{2 \omega_{n} \varepsilon} \geq \alpha
$$

Notice that since $\operatorname{Ecc}_{E}^{v}\left(x_{0}, \rho\right)=0$, we have

$$
P\left(E, B_{\rho}\left(x_{0}\right)\right)=\int_{B_{\rho}\left(x_{0}\right)}\left\langle\nu_{E}(y), v\right\rangle \mathrm{d}\left\|\bar{D} \chi_{E}\right\|(y),
$$

which means that $\nu_{E}(y) \in v^{*}$ for $\left\|\bar{D} \chi_{E}\right\|$-a.e. $y \in B_{\rho}\left(x_{0}\right)$. Hence, since $\mu_{\varepsilon} / \alpha_{\varepsilon}$ are probability measures and $v^{*}$ is a convex set, we obtain

$$
\bar{\nabla} u_{\varepsilon}(x)=\left(\bar{D} \chi_{E} * \varphi_{\varepsilon}\right)(x)=\alpha_{\varepsilon} \int \nu_{E}(y) \mathrm{d} \frac{\mu_{\varepsilon}}{\alpha_{\varepsilon}}(y) \in \alpha_{\varepsilon} v^{*}
$$


We have just proved that if $u_{\varepsilon}(x)=1 / 2\left(\right.$ with $\left.B_{\varepsilon}(x) \subseteq B_{\rho}\left(x_{0}\right)\right)$ then $\bar{D} u_{\varepsilon}(x) \in \alpha_{\varepsilon} v^{*}$ with $\alpha_{\varepsilon} \geq \alpha$, which means that $\partial u_{\varepsilon} / \partial v(x)<0$ as $\langle\xi, v\rangle>0$ for all $\xi \in v^{*}$. Thus we may apply the implicit function theorem to obtain that $\left\{u_{\varepsilon}(x)=1 / 2\right\} \cap B_{\rho-\varepsilon}\left(x_{0}\right)$ is contained in the graph of a $\mathcal{C}^{\infty}$ function $f_{\varepsilon}: T \rightarrow \mathbb{R} v$. Since $\varepsilon\left\|\nabla u_{\varepsilon}\right\|$ is bounded from above in $X$ and $\varepsilon \partial u^{\varepsilon} / \partial v$ is bounded from below in $\left\{u_{\varepsilon}=1 / 2\right\}$ we obtain that

$$
\frac{\partial f_{\varepsilon}}{\partial z}=-\left(\frac{\partial u_{\varepsilon}}{\partial v}\right)^{-1}\left(\frac{\partial u_{\varepsilon}}{\partial z}\right) \quad z \in T \cap \partial B_{1}
$$

is bounded, hence $f_{\varepsilon}$ are $L_{0}$-lipschitz for some constant $L_{0}$ independent of $\varepsilon$.

Since $u_{\varepsilon} \rightarrow \chi_{E}$ in $L^{1}$, letting $E_{\varepsilon}:=\left\{u_{\varepsilon} \geq 1 / 2\right\}$ we have $\left|E_{\varepsilon} \triangle E\right| \rightarrow 0$. Recalling that $\left\|D f_{\varepsilon}\right\|$ are equi-bounded, we have

$$
|| f_{\varepsilon_{1}}-f_{\varepsilon_{2}} \|_{\mathcal{C}^{0}} \leq C|| f_{\varepsilon_{1}}-\left.f_{\varepsilon_{2}}\right|_{L^{1}}=C\left|E_{\varepsilon_{1}} \triangle E_{\varepsilon_{2}}\right|
$$

for some dimensional constant $C$, so $f_{\varepsilon} \rightarrow f$ uniformly and since $f_{\varepsilon}$ are all $L_{0}$-lipschitz also $f$ is $L_{0}$-lipschitz and $E \cap B_{\rho}\left(x_{0}\right)$ is the subgraph of $f$ along $v$.

Proposition 4.2. The best constant $L_{0}$ in the previous theorem, valid for any $v \in X$ and any hyper-space $T$ perpendicular to $v$, is given by

$$
L_{0}=\sup _{\|v\|=1} \sup _{\xi, \nu \in v^{*}} \sup _{z \in \xi^{\perp},\|z\|=1}|\langle z, \nu\rangle|
$$

In particular, $L_{0}=0$ if $B_{1}^{*}$ is strictly convex (since in this case $\xi=\nu$ in the previous formula).

Proof. Given any $v \in X$ with $\|v\|=1$ the sets $E$ such that $\operatorname{Ecc}_{E}^{v}(x, \rho)=0$ are exactly those for which $\nu_{E} \in v^{*}$ $\left\|\bar{D} \chi_{E}\right\|$-a.e. in $B_{\rho}(x)$. So, given $\xi \in v^{*}$ and $T=\xi^{\perp}$, if $f$ is the lipschitz function given by the previous lemma then we have $D f(z) h=-\left\langle h, \nu_{E}(z+f(z))\right\rangle v$ (this can be easily proven noticing that $\left\{h+t v: h \in \xi^{\perp}, t v \leq\right.$ $\left.D f(z) h\}=\left\{h+t v:\left\langle h+t v, \nu_{E}(z+f(z))\right\rangle \leq 0\right\}\right)$. So the lipschitz constant of $D f(z)$ is given by

$$
\sup _{\|h\|=1}\|D f(z) h\|=\sup _{\|h\|=1}|\langle h, \nu\rangle| .
$$

The conclusion follows considering all possible $v, \xi$ and $\nu$.

Proposition 4.3 (properties of the excess).

1. If $B_{\rho}(x) \subset B_{\eta}(y)$, then

$$
\begin{aligned}
& \operatorname{Ecc}_{E}^{v}(x, \rho) \leq\left(\frac{\eta}{\rho}\right)^{n-1} \operatorname{Ecc}_{E}^{v}(y, \eta) \\
& \operatorname{Ecc}_{E}(x, \rho) \leq\left(\frac{\eta}{\rho}\right)^{n-1} \operatorname{Ecc}_{E}(y, \eta)
\end{aligned}
$$

2. The functions $\rho \mapsto \mathbf{E c c}_{E}(x, \rho)$ and $\rho \mapsto \mathbf{E c c}_{E}^{v}(x, \rho)$ are left-continuous in $] 0,+\infty[$.

3. Let $\left(E_{k}\right)$ be a sequence of $\omega$-minimal sets converging to $E$ in $L_{\mathrm{loc}}^{1}(X)$ and $v_{k} \rightarrow v$. Then

$$
\begin{aligned}
& \lim _{k \rightarrow \infty} \operatorname{Ecc}_{E_{k}}(x, \rho)=\operatorname{Ecc}_{E}(x, \rho) \\
& \lim _{k \rightarrow \infty} \mathbf{E c c}_{E_{k}}^{v_{k}}(x, \rho)=\operatorname{Ecc}_{E}^{v}(x, \rho)
\end{aligned}
$$


for all $x \in X$ and all $\rho>0$ such that $P\left(E, \partial B_{\rho}(x)\right)=0$. While

$$
\begin{aligned}
\liminf _{k \rightarrow \infty} \mathbf{E c c}_{E_{k}}(x, \rho) & \geq \mathbf{E c c}_{E}(x, \rho) \\
\liminf _{k \rightarrow \infty} \mathbf{E c c}_{E_{k}}^{v_{k}}(x, \rho) & \geq \mathbf{E c c}_{E}^{v}(x, \rho),
\end{aligned}
$$

for all $x \in X$ and all $\rho>0$.

4. If $\operatorname{Ecc}_{E}(x, \rho)=0$ then $E \in \mathcal{M}_{0}\left(B_{\rho}(x)\right)$.

Proof. 1. Note that

$$
\rho^{n-1} \operatorname{Eccc}_{E}^{v}(x, \rho)=\int_{B_{\rho}(x)}\left(1-\left\langle\nu_{E}(y), v\right\rangle\right) \mathrm{d}\left\|\bar{D} \chi_{E}\right\|(y)
$$

Since the integrand is always nonnegative, we can conclude that $\rho^{n-1} \mathbf{E c c c}_{E}^{v}(x, \rho) \leq \eta^{n-1} \mathbf{E c c}_{E}^{v}(y, \eta)$. Moreover, if $\mathbf{E c c}_{E}(x, \rho)=\mathbf{E c c}_{E}^{v}(x, \rho)$ and $\mathbf{E c c}_{E}(y, \eta)=\mathbf{E c c}_{E}^{w}(y, \eta)$, then

$$
\mathbf{E c c}_{E}(x, \rho)=\mathbf{E c c}_{E}^{v}(x, \rho) \leq \mathbf{E c c}_{E}^{w}(x, \rho) \leq\left(\frac{\eta}{\rho}\right)^{n-1} \operatorname{Ecc}_{E}^{w}(y, \eta)=\left(\frac{\eta}{\rho}\right)^{n-1} \operatorname{Ecc}_{E}(y, \eta)
$$

2. It follows immediately from the definitions of $\mathbf{E c c}_{E}^{v}$ and $\mathbf{E c c}_{E}$.

3. As $D \chi_{E_{k}} \stackrel{*}{\rightarrow} D \chi_{E}$ and $\left\|\bar{D} \chi_{E_{k}}\right\| \stackrel{*}{\rightarrow}\left\|\bar{D} \chi_{E}\right\|$, the first statement follows. Given $x \in X$ and $\rho>0$, choose $\rho^{\prime}<\rho$ such that $P\left(E, \partial B_{\rho^{\prime}}(x)\right)=0$. Then, we have

$$
\begin{aligned}
& \left(\rho^{\prime}\right)^{n-1} \mathbf{E c c}_{E}\left(x, \rho^{\prime}\right)=\lim _{h \rightarrow \infty}\left(\rho^{\prime}\right)^{n-1} \mathbf{E}_{\mathbf{c c}_{E_{h}}}\left(x, \rho^{\prime}\right) \leq \liminf _{h \rightarrow \infty} \rho^{n-1} \mathbf{E c c}_{E_{h}}(x, \rho), \\
& \left(\rho^{\prime}\right)^{n-1} \mathbf{E c c c}_{E}^{v}\left(x, \rho^{\prime}\right)=\lim _{h \rightarrow \infty}\left(\rho^{\prime}\right)^{n-1} \mathbf{E}_{\mathbf{c c}_{E_{h}}^{v}}\left(x, \rho^{\prime}\right) \leq \liminf _{h \rightarrow \infty} \rho^{n-1} \mathbf{E}_{\mathbf{c c}_{E_{h}}^{v}}(x, \rho) .
\end{aligned}
$$

One gets the thesis using property 1 and letting $\rho^{\prime} \rightarrow \rho$.

4. Given any $F$ such that $E \triangle F \Subset B_{\rho}(x)$ by Proposition 3.10 we obtain

$$
P\left(E, B_{\rho}(x)\right)=\left\|\bar{D} \chi_{E}\left(B_{\rho}(x)\right)\right\|=\left\|\bar{D} \chi_{F}\left(B_{\rho}(x)\right)\right\| \leq P\left(F, B_{\rho}(x)\right)
$$

Lemma 4.4 (vertical gap). For any $L>L_{0}$ there exists $\varepsilon_{1}=\varepsilon_{1}(n,\|\cdot\|, \omega, L)>0$ such that the following property holds. Let $E \in \mathcal{M}_{\omega}\left(B_{3 \rho}\right), \omega(\rho) \leq 1, v \in X \cap \partial B_{1}$ and let $T \subset X$ be an hyper-space perpendicular to $v$. Let $x=z+t v, x^{\prime}=z^{\prime}+t^{\prime} v$ be two points of $B_{\rho} \cap \partial E$ with $z, z^{\prime} \in T$ and $t, t^{\prime} \in \mathbb{R}$. If we in addition suppose that

$$
\left.\left.\operatorname{Ecc}_{E}^{v}(w, \eta) \leq \varepsilon_{1} \quad \forall w \in\left\{x, x^{\prime}\right\} \subset B_{\rho}\left(x_{0}\right) \cap \partial E, \eta \in\right] 0,2 \rho\right]
$$

we conclude that

$$
\left\|t v-t^{\prime} v\right\| \leq L\left\|z-z^{\prime}\right\|
$$

Proof. Without loss of generality we may suppose that $\rho=1$. Suppose also, by contradiction, that there exist $\omega$-minimal sets $E_{k}$ in $B_{3}, v_{k} \in \partial B_{1}$, hyper-planes $T_{k}$ and points $x_{k}=z_{k}+t_{k} v_{k}, x_{k}^{\prime}=z_{k}^{\prime}+t_{k}^{\prime} v_{k}$ with $x_{k}, x_{k}^{\prime} \in B_{1} \cap \partial E_{k}, z_{k}, z_{k}^{\prime} \in T_{k}$ and $t_{k}, t_{k}^{\prime} \in \mathbb{R}$ such that

$$
\mathbf{E c c c}_{E_{k}}^{v_{k}}(w, \eta) \leq \frac{1}{k}
$$


for all $\eta \in(0,2], w \in\left\{x_{k}, x_{k}^{\prime}\right\}$ and

$$
\left\|t_{k} v-t_{k}^{\prime} v\right\|>L\left\|z_{k}-z_{k}^{\prime}\right\| .
$$

Case 1. Suppose that $\liminf _{k \rightarrow \infty}\left|t_{k}-t_{k}^{\prime}\right|>0$. Up to a subsequence we may suppose that $t_{k} \rightarrow t, t_{k}^{\prime} \rightarrow t^{\prime} \neq t$, $v_{k} \rightarrow v, T_{k} \rightarrow T, x_{k} \rightarrow x=z+t v, x_{k}^{\prime} \rightarrow x^{\prime}=x^{\prime}+t^{\prime} v$ and by Proposition $3.3 E_{k} \rightarrow E$. Now for $k$ sufficiently large we have

$$
\mathbf{E c c}_{E_{k}}^{v_{k}}(x, 3 / 2) \leq \frac{4^{n-1}}{3^{n-1}} \mathbf{E c c}_{E_{k}}^{v_{k}}\left(x_{k}, 2\right) \leq \frac{4^{n-1}}{3^{n-1} k} .
$$

By Proposition 4.3 we get $\mathbf{E c c}_{E}^{v}(x, 3 / 2)=0$ and similarly $\mathbf{E c c}_{E}^{v}\left(x^{\prime}, 3 / 2\right)=0$. So by Lemma 4.1 we know that in the union $B_{3 / 2}(x) \cup B_{3 / 2}\left(x^{\prime}\right)$ the set $\partial E$ coincides with the graph along $v$ of a $L_{0}$-lipschitz function $f: T \rightarrow \mathbb{R} v$. But $f(z)=t v$ and $f\left(x^{\prime}\right)=t^{\prime} v$ and since we have assumed $\left\|t v-t^{\prime} v\right\|>L\left\|z-z^{\prime}\right\|$ we get a contradiction when $L>L_{0}$.

Case 2. Suppose that $\liminf _{k \rightarrow \infty}\left|t_{k}-t_{k}^{\prime}\right|=0$ and let $\eta_{k}:=\left\|t_{k} v-t_{k}^{\prime} v\right\|$. Up to a subsequence we may suppose that $\eta_{k} \rightarrow 0$. Define $F_{k}:=\left(E_{k}-x_{k}\right) / \eta_{k}$ and $y_{k}=\left(x_{k}-x_{k}^{\prime}\right) / \eta_{k}$. We get

$$
\left\|y_{k}\right\| \leq \frac{\left\|z_{k}-z_{k}^{\prime}\right\|+\left\|t_{k} v-t_{k}^{\prime} v\right\|}{\eta_{k}} \leq 1+\frac{1}{L},
$$

so, up to a subsequence, we may suppose $y_{k} \rightarrow y=z \pm v /\| \pm v\|$ for some $z \in T$. By the hypothesis $\left\|t_{k} v-t_{k}^{\prime} v\right\|>L\left\|z_{k}-z_{k}^{\prime}\right\|$ we get $\|z\|<1 / L$. Let $R:=1+1 / L$. Again, up to a subsequence, we may suppose that $F_{k} \rightarrow F$ and that $R \eta_{k}<2$. The hypothesis $\mathbf{E c c c}_{E_{k}}^{v_{k}}\left(w, R \eta_{k}\right) \leq 1 / k$ for $w \in\left\{x_{k}, x_{k}^{\prime}\right\}$ becomes $\operatorname{Ecc}_{F_{k}}^{v_{k}}(0, R) \leq 1 / k$ and $\mathbf{E c c}_{F_{k}}^{v_{k}}\left(y_{k}, R\right) \leq 1 / k$, so that $\mathbf{E c c}_{F_{k}}^{v_{k}}(y, R / 2) \leq 2^{n-1} / k$, and by Proposition 4.3 we conclude

$$
\mathbf{E c c}_{F}^{v}(0, R)=\mathbf{E c c}_{F}^{v}(0, R / 2)=0 .
$$

As in Case 1 we note that the set $\partial F$ must be a graph along $v$ of an $L_{0}$-lipschitz function $f$ defined on $T$, but again we note that since $f(0)=0$ and $f(z)= \pm 1$ the lipschitz constant of $f$ is at least $L$. This contradicts $L>L_{0}$.

Lemma 4.5 (horizontal translations). There exist positive constants $c_{0}$, $\varepsilon_{0}$ depending on $(n,\|\cdot\|, \omega)$ such that, given any $E \in \mathcal{M}_{\omega}\left(B_{2\left(1+L_{0}\right) \rho}\left(x_{0}\right)\right), \omega(\rho) \leq 1$, and given an hyper-space $T$ and a unit vector $v$ perpendicular to $T$, then for all $h \in T \cap B_{\rho}$ there holds

$$
\operatorname{Ecc}_{E}^{v}\left(x_{0}, 2\left(1+L_{0}\right) \rho\right)<\varepsilon_{0} \Rightarrow \mathcal{H}^{n-1}\left(\partial E \cap C\left(x_{0}, h, \rho\right)\right) \geq c_{0} \rho^{n-1},
$$

where

$$
C\left(x_{0}, h, \rho\right)=\left\{x_{0}+z+t v: z \in B_{\rho}(h) \cap T,\|t v\| \leq 2 L_{0} \rho\right\} .
$$

Proof. Suppose for simplicity $x_{0}=0, \rho=1$ and let $C(h):=C(0, h, 1)$. Reasoning by contradiction we suppose that there exist $E_{k} \in \mathcal{M}_{\omega}\left(B_{2\left(1+L_{0}\right)}\right)$, hyper-spaces $T_{k}$, unit vectors $v_{k}$ perpendicular to $T_{k}$ and $h_{k} \in T_{k} \cap B_{1}$ such that

$$
\operatorname{Eccc}_{E_{k}}^{v_{k}}\left(0,2\left(1+L_{0}\right)\right)<\frac{1}{k}, \quad \text { and } \quad \mathcal{H}^{n-1}\left(\partial E \cap C\left(h_{k}\right)\right)<c_{0} .
$$

Up to a subsequence we may also suppose that $E_{k} \rightarrow E, v_{k} \rightarrow v, T_{k} \rightarrow T, h_{k} \rightarrow h$, so that $\mathbf{E c c}_{E}^{v}\left(0,2\left(1+L_{0}\right)\right)=0$ and $\mathcal{H}^{n-1}(\partial E \cap C(h))<c_{0}$. So by Lemma 4.1, in $B_{2\left(1+L_{0}\right)} \partial E$ is the graph over $T$ of an $L_{0}$-lipschitz function $f: T \rightarrow \mathbb{R} v$. Since $C(h) \subset B_{2\left(1+L_{0}\right)}$ we get $\partial E \cap C(h)=\Gamma_{f} \cap C(h)$. Moreover, if $\pi: X \rightarrow T$ is the projection on $T$ along $v(\pi(z+t v):=z$ for all $z \in T, t \in \mathbb{R})$, it is not difficult to show that $z \in \pi(C(h)) \Rightarrow z+f(z) \in C(h)$ which means $\pi\left(\Gamma_{f} \cap C(h)\right)=\pi(C(h))=B_{1}(h)$. Finally we conclude that $\mathcal{H}^{n-1}(\partial E \cap C(h)) \geq K \mathcal{H}^{n-1}\left(T \cap B_{1}(h)\right)$, where $K$ is the lipschitz constant of $\pi$, so that for $c_{0}$ sufficiently small we get a contradiction. 
In the following proposition, we will show that the boundary of a quasi minimizer $E$ can be locally approximated by the graph $\Gamma$ of a lipschitz function, estimating the measure of $\partial E \triangle \Gamma$ with the excess.

Proposition 4.6 (lipschitz approximation). For each $L>L_{0}$ there exists a constant $c(L)>0$ such that, given any unit vector $v \in X \cap B_{1}$ and any hyper-space $T \subset$ perpendicular to $v$, for any $E$ in $\mathcal{M}_{\omega}(\Omega), x_{0} \in \partial E$ and for all $\rho>0$ sufficiently small (more precisely $9\left(2 L_{0}+1\right) \rho<\rho_{\omega}, \omega\left(\left(2 L_{0}+1\right) \rho\right)<1$ and $\left.B_{9\left(2 L_{0}+1\right) \rho}\left(x_{0}\right) \subset \Omega\right)$ the following statements are true.

1. If $\mathbf{E}_{\mathbf{c c}}^{v}(x, \eta) \leq \varepsilon_{1}(L)$ (where $\varepsilon_{1}(L)$ is the constant given in Lemma 4.4) for all $x \in \partial E \cap \overline{B_{\rho}\left(x_{0}\right)}$ and for all $\eta \in] 0,2 \rho\left[\right.$, then $\partial E \cap B_{\rho}\left(x_{0}\right)$ is contained in the graph along $v$ of a L-lipschitz function $f: T \rightarrow \mathbb{R} v$.

2. There exists a L-lipschitz function $f: T \rightarrow \mathbb{R} v$ such that

$$
\mathcal{H}^{n-1}\left(\left(\partial E \triangle \Gamma_{f}\right) \cap B_{\rho}\left(x_{0}\right)\right) \leq c(L) \rho^{n-1} \operatorname{Eccc}_{E}^{v}\left(x_{0}, 9\left(2 L_{0}+1\right) \rho\right) .
$$

Proof. Suppose for simplicity $x_{0}=0$.

Step 1. Define

$$
G:=\left\{x \in \overline{\partial E \cap B_{\rho}}: \mathbf{E c c c}_{E}^{v}(x, \eta) \leq \varepsilon_{1}(L) \quad \forall \eta \in\right] 0,2 \rho[\}
$$

From Lemma 4.4 we know that given any two points $x=z+t v$ and $x^{\prime}=z^{\prime}+t^{\prime} v$ of $G$ we have $\left|t-t^{\prime}\right| \leq L|| z-z^{\prime}||$. It follows that the projection $\pi$ of $G$ on $T$ along $v$ is injective and $G$ is the graph of a $L$-lipschitz function $f$ which can be extended to all $T$. So the first statement is easily proved, since in that case $G=\partial E \cap \overline{B_{\rho}}$.

Step 2. Let now

$$
G^{\prime}:=\left\{x \in \overline{B_{3\left(2 L_{0}+1\right) \rho}} \cap \partial E: \mathbf{E c c}_{E}^{v}(x, \eta) \leq \varepsilon \quad \forall \eta \in\left(0,6\left(2 L_{0}+1\right) \rho\right)\right\}
$$

where $\varepsilon>0$ is such that $\varepsilon<\varepsilon_{0}$ and $\varepsilon<\varepsilon_{1}(L)$.

Now we will estimate the measure of the set $U:=\partial E \cap B_{3\left(2 L_{0}+1\right) \rho} \backslash G^{\prime}$. For all $x \in U$ there exists $\rho_{x} \in\left(0,6\left(2 L_{0}+1\right) \rho\right)$ such that $\mathbf{E c c}_{E}^{v}\left(x, \rho_{x}\right)>\varepsilon$, By Besicovitch covering theorem we can find a countable covering $\left\{B_{\rho_{i}}\left(x_{i}\right)\right\}$ of $U\left(\rho_{i}:=\rho_{x_{i}}\right)$, with the property that for all $x \in U$ there are at most $N$ balls $B_{\rho_{i}}\left(x_{i}\right)$ which contain $x(N$ is a constant depending only on $(X,\|\cdot\|))$. So we get

$$
\begin{aligned}
\mathcal{H}^{n-1}(U) & \leq \sum_{i} \mathcal{H}^{n-1}\left(\partial E \cap B_{\rho_{i}}\left(x_{i}\right)\right) \leq K \sum_{i} \rho_{i}^{n-1} \\
& \leq \frac{K}{\varepsilon} \sum_{i} \rho_{i}^{n-1} \operatorname{Eccc}_{E}^{v}\left(x_{i}, \rho_{i}\right) \leq \frac{K N}{\varepsilon}\left(9\left(2 L_{0}+1\right) \rho\right)^{n-1} \mathbf{E c c c}_{E}^{v}\left(0,9\left(2 L_{0}+1\right) \rho\right)
\end{aligned}
$$

Step 3. Let $f$ be the $L$-lipschitz function, defined as in Step 1 , such that $G^{\prime} \subseteq \Gamma_{f}$. Also we can suppose $L<5 L_{0} / 4$. We will now estimate the measure of the set $V:=\left(\Gamma_{f} \cap B_{\rho}\right) \backslash \partial E$. Let $\pi(V)$ be the projection of $V$ on $T$ along $v$. By (10), it is enough to estimate from above the $(n-1)$-dimensional measure of $\pi(V)$ with a multiple of $\mathcal{H}^{n-1}(U)$. Observe that we can assume $G^{\prime} \cap\left(-B_{\frac{\rho}{3}}\right) \neq \emptyset$, indeed if not it would follow $\mathcal{H}^{n-1}(U) \geq$ $\mathcal{H}^{n-1}\left(\partial E \cap\left(-B_{\frac{\rho}{3}}\right)\right) \geq c(\rho / 3)^{n-1}$, by Proposition 3.5; therefore, by (10) we would have $\mathbf{E c c}_{E}^{v}\left(0,9\left(2 L_{0}+1\right) \rho\right) \geq k \varepsilon$, for some $k>0$, so that choosing $c(L)$ large enough, $T$ itself satisfies the thesis. So we assume $G^{\prime} \cap B_{\frac{\rho}{3}} \neq \emptyset$. Let $z \in \pi(V)$ and let $C_{r}(z):=\left(B_{r}(z) \cap T\right) \oplus \mathbb{R} v$ be the largest cylinder not intersecting $G^{\prime}$. We have $r<4 \rho / 3$, moreover there exists $z^{\prime} \in T \cap \partial B_{r}(z)$ such that $z^{\prime}+f\left(z^{\prime}\right) \in G^{\prime}$. By Lemma 4.5 with $h=z^{\prime}-z$, we get $\mathcal{H}^{n-1}\left(\partial E \cap C_{r}^{v}\right) \geq c_{0} r^{n-1}$, where $C_{r}^{v}:=C\left(z+f\left(z^{\prime}\right), z^{\prime}-z, r\right)$. Since $\left\|z+f\left(z^{\prime}\right)\right\| \leq \rho(4+8 L) / 3$, it follows that, for $L<5 L_{0} / 4$ we have $\partial E \cap C_{r}^{v} \subset U \cap C_{r}(z)$, which in turn implies $\mathcal{H}^{n-1}\left(U \cap C_{r}(z)\right) \geq c_{0} r^{n-1}$. As before, we can find a collection of balls $\left\{B_{r_{i}}\left(z_{i}\right)\right\}, z_{i} \in T$, such that $N \mathcal{H}^{n-1}\left(\bigcup_{i} B_{r_{i}}\left(z_{i}\right) \cap T\right) \geq \mathcal{H}^{n-1}(\pi(V))$ and $\mathcal{H}^{n-1}\left(U \cap C_{r_{i}}\left(z_{i}\right)\right) \geq c_{0} r_{i}^{n-1}$. Summing up over $i$ we get $\mathcal{H}^{n-1}(\pi(V)) \leq M \mathcal{H}^{n-1}(U)$, for a suitable constant $M>0$. 
We conclude this section recalling the following lemma, that we will be needed in the sequel (see [5], Lem. 4.4.5).

Lemma 4.7. Let $E \subset X$ be a set of finite perimeter in $\Omega$, where $\Omega$ is an open connected subset of $X$. If $\partial E \cap \Omega$ is non empty and is contained in a lipschitz graph $\Gamma$, then $\partial E \cap \Omega=\Gamma \cap \Omega$.

\section{The Uniformly CONVEX CASE} have

We say that the ball $B_{1}^{*}$ is uniformly convex if there exists a constant $M>0$ such that for all $\xi, \eta \in \partial B_{1}^{*}$ we

$$
\|\xi-\eta\|_{s}^{2} \leq M\left(1-\frac{\|\xi+\eta\|}{2}\right)
$$

For any set $E$ with locally finite perimeter in $\Omega$ and any ball $B_{\rho} \Subset \Omega$ we define the approximate normal $\nu_{E}(x, \rho)$ by

$$
\nu_{E}(x, \rho):=\frac{\bar{D} \chi_{E}\left(B_{\rho}(x)\right)}{\left\|\bar{D} \chi_{E}\left(B_{\rho}(x)\right)\right\|}
$$

Proposition 5.1. Assume that $B_{1}^{*}$ is uniformly convex and let $E \in \mathcal{M}_{\omega}(\Omega)$ be such that $\mathbf{E c c}_{E}(x, \rho) \leq C \rho^{2 \alpha}$ for all balls $B_{\rho}(x) \subset \Omega, x \in \partial E$, for some constant $C, \alpha>0$. Then $\partial^{*} E=\partial E$ in $\Omega$ and $\nu_{E}(x): \partial E \cap \Omega \rightarrow X^{*}$ is $\alpha$-Hölder continuous. Hence, $\partial E \cap \Omega$ is an hyper-surface of class $\mathcal{C}^{1, \alpha}$.

Proof. From the lower density estimate and the upper bound on the excess we get

$$
\left.\left\|\bar{D} \chi_{E}\left(B_{\rho}(x)\right)\right\| \geq 2 \theta \rho^{n-1} \quad \forall B_{\rho}(x) \subset \Omega, \rho \in\right] 0, \rho_{0}[,
$$

for a suitable constant $\rho_{0}$.

Let $B_{\rho}(x) \subset B_{\eta}(y) \subset \Omega$ with $x, y \in \partial E$ and $\eta<\rho_{0}$; for any $w \in \nu_{E}(y, \eta)^{*}$ we obtain

$$
\begin{aligned}
2\left\|\nu_{E}(x, \rho)-\nu_{E}(y, \eta)\right\|_{s}^{2} & \leq M\left(2-\left\|\nu_{E}(x, \rho)+\nu_{E}(y, \eta)\right\|\right) \leq M\left(2-\left\langle\nu_{E}(x, \rho)+\nu_{E}(y, \eta), w\right\rangle\right) \\
& =M\left(1-\left\langle\nu_{E}(x, \rho), w\right\rangle\right) \leq M\left(\frac{\left\|\bar{D} \chi_{E}\right\|\left(B_{\rho}(x)\right)}{\left\|\bar{D} \chi_{E}\left(B_{\rho}(x)\right)\right\|}-\left\langle\nu_{E}(x, \rho), w\right\rangle\right) \\
& =M \rho^{n-1} \frac{\operatorname{Eccc}_{E}^{w}(x, \rho)}{\left\|\bar{D} \chi_{E}\left(B_{\rho}(x)\right)\right\|} \leq \frac{M}{2 \theta}\left(\frac{\eta}{\rho}\right)^{n-1} \operatorname{Eccc}_{E}^{w}(y, \eta) .
\end{aligned}
$$

Since $w \in \nu_{E}(y, \eta)^{*}$ we have $\operatorname{Ecc}_{E}(y, \eta)=\operatorname{Eccc}_{E}^{w}(y, \eta)$, hence

$$
2\left\|\nu_{E}(x, \rho)-\nu_{E}(y, \eta)\right\|_{s}^{2} \leq \frac{M}{2 \theta}\left(\frac{\eta}{\rho}\right)^{n-1} \operatorname{Ecc}_{E}(y, \eta) \leq \frac{M C}{2 \theta}\left(\frac{\eta}{\rho}\right)^{n-1} \eta^{2 \alpha}
$$

Let now $x \in \partial^{*} E$. Since $\lim _{\rho \rightarrow 0}\left\|\nu_{E}(x, \rho)-\nu_{E}(x)\right\|=0$, for $\rho<\rho_{0}$ we obtain

$$
\begin{aligned}
\left\|\nu_{E}(x)-\nu_{E}(x, \rho)\right\|_{s} & \leq \sum_{k=0}^{\infty}\left\|\nu_{E}\left(x, 2^{-k} \rho\right)-\nu_{E}\left(x, 2^{-(k+1)} \rho\right)\right\|_{s} \\
& \leq C^{\prime} \rho^{\alpha}
\end{aligned}
$$


and a similar estimate proves that $\nu_{E}$ exists for any $x \in \partial E$, i.e. $\partial^{*} E=\partial E$. Let $x, y \in \partial E \cap \Omega, \rho=\|x-y\|_{s}$, $z=(x+y) / 2$ and $\eta=2 \rho$. We have

$$
\begin{aligned}
\left\|\nu_{E}(x)-\nu_{E}(y)\right\|_{s} \leq & \left\|\nu_{E}(x)-\nu_{E}(x, \rho)\right\|_{s}+\left\|\nu_{E}(y)-\nu_{E}(y, \rho)\right\|_{s} \\
& +\left\|\nu_{E}(x, \rho)-\nu_{E}(z, \eta)\right\|_{s}+\left\|\nu_{E}(y, \rho)-\nu_{E}(z, \eta)\right\|_{s} \\
\leq & C^{\prime \prime} \rho^{\alpha}
\end{aligned}
$$

Fix now $x_{0} \in \partial E, B_{\rho}\left(x_{0}\right) \subset \Omega$. For $\rho$ sufficiently small, by Proposition 4.6, there exists a lipschitz function $f: T \rightarrow \mathbb{R}$ such that $\partial E \cap B_{\rho}\left(x_{0}\right) \subset \Gamma_{f}$. We want to prove that $f$ is of class $\mathcal{C}^{1, \alpha}$. Let $x=(z, t), \pi: X \rightarrow T$ the projection and $A:=\pi\left(B_{\rho}\left(x_{0}\right) \cap \Gamma_{f}\right)$, which is relatively open in $T$. Let also $D:=\{z \in A: \exists \nabla f(z),(z, f(z)) \in$ $\left.\partial^{*} E\right\}$. Then $\mathcal{H}^{n-1}(A \backslash D)=0$ and the function $z \in D \rightarrow \nabla f(x)$ is $\alpha$-Hölder continuous by (11). It follows that $f \in \mathcal{C}^{1, \alpha}(A)$.

\section{Regularity in the two-Dimensional CASE}

In this section we consider the case $n=2$. In this case the boundary of a set $E \in \mathcal{M}_{\omega}(\Omega)$ can be directly compared with straight lines. This simple fact enable us to get a decay result (Lem. 6.2) for the excess of $\omega$-minimal sets.

Lemma 6.1. For any $L>0$ there exist $\left.\rho_{0}=\rho_{0}(L) \in\right] 0, \rho_{\omega}[$ and $\varepsilon=\varepsilon(L)>0$ such that, given E $\in$ $\mathcal{M}_{\omega}\left(B_{\rho}\left(x_{0}\right)\right), x_{0} \in \partial E, 0<\rho<\rho_{0}$ and a L-lipschitz function $f: T \rightarrow \mathbb{R} v$, with $v$ unit vector perpendicular to the hyper-space $T$, the following holds. If

$$
\mathcal{H}^{1}\left(\left(\partial E \triangle \Gamma_{f}\right) \cap B_{\rho}\left(x_{0}\right)\right) \leq \varepsilon \rho
$$

then there exists a convex open set $R$ with $B_{\rho /(6+4 L)}\left(x_{0}\right) \subset R \subset B_{\rho}\left(x_{0}\right)$ such that $\partial E \cap \partial R$ consist of exactly two points. Moreover, $\bar{E} \cap \partial R$ and $\overline{X \backslash E} \cap \partial R$ are connected.

Proof. Suppose for simplicity $x_{0}=0$. Let $\pi: X \rightarrow T$ and $\pi^{\prime}: X \rightarrow \mathbb{R} v$ be defined respectively by $\pi(z+t v)=z$ and $\pi^{\prime}(z+t v)=t v$ for all $z \in T$ and $t \in \mathbb{R}$. Consider the set $D:=\left\{z \in T: \#\left\{\pi^{-1}(z) \cap \partial E \cap B_{\rho}\right\} \neq 1\right\}$. Clearly $D=\pi\left(\pi^{-1}(D) \cap\left(\partial E \triangle \Gamma_{f}\right) \cap B_{\rho}\right)$, since $\#\left\{\pi^{-1}(z) \cap \Gamma_{f}\right\}=1$. So, letting $K$ be a lipschitz constant for $\pi$ (chosen independent of $T$ and $v)$, we have

$$
\mathcal{H}^{1}(D) \leq K \mathcal{H}^{1}\left(\left(\partial E \triangle \Gamma_{f}\right) \cap B_{\rho}\right) \leq K \varepsilon \rho
$$

Set $\eta=\rho /(3+2 L)$ and let $\varepsilon$ be so small that $K \varepsilon \rho \leq \eta / 3$. As $\mathcal{H}^{1}\left(T \cap B_{\eta} \backslash B_{\eta / 2}\right) \geq \eta$ there exist two points $z_{1}, z_{2} \in\left(T \cap B_{\eta} \backslash B_{\eta / 2}\right) \backslash D$ such that $T \cap B_{\eta / 2} \subset\left[z_{1}, z_{2}\right] \subset T \cap B_{\eta}$.

Now notice that $\pi^{\prime}$ is also lipschitz since $v$ is perpendicular to $T$ and denote by $K^{\prime}$ its lipschitz constant. Define $D^{\prime}=\pi^{\prime}\left(\partial E \cap B_{\rho} \cap \pi^{-1}(] z_{1}, z_{2}[)\right)$. Note that for $\varepsilon$ sufficiently small there holds $\Gamma_{f} \cap B_{\eta} \neq \emptyset$ otherwise we had $\partial E \cap B_{\eta} \subset \partial E \cap B_{\rho} \backslash \Gamma_{f}$ but by Proposition $3.5 \mathcal{H}^{1}\left(\partial E \cap B_{\eta}\right) \geq \theta^{\prime} \eta$ for some $\theta^{\prime}>0$. It follows that $\pi^{\prime}\left(\Gamma_{f} \cap \pi^{-1}\left(B_{\eta} \cap T\right)\right) \subset B_{(1+2 L) \eta} \cap \mathbb{R} v$. Since $D^{\prime} \backslash B_{(1+2 L) \eta} \subset \pi^{\prime}\left(\partial E \cap B_{\rho} \backslash \Gamma_{f}\right)$ we obtain $\mathcal{H}^{1}\left(D^{\prime} \backslash B_{(1+2 L) \eta}\right) \leq$ $K^{\prime} \varepsilon \rho \leq \frac{1}{3} \eta$ if $\varepsilon$ is sufficiently small. But $\mathcal{H}^{1}\left(B_{(2+2 L) \eta} \cap \mathbb{R} v \backslash B_{(1+2 L) \eta}\right) \geq \eta$ so it is possible to find two points $t_{1} v, t_{2} v \in \mathbb{R} v \backslash D^{\prime}$ such that $B_{(1+2 L) \eta} \cap \mathbb{R} v \subset\left[t_{1} v, t_{2} v\right] \subset B_{(2+2 L) \eta} \cap \mathbb{R} v$.

Define $R=] z_{1}, z_{2}[\oplus] t_{1} v, t_{2} v$ [ and notice that $R \subset B_{\rho}$ and $R \supset B_{\eta / 2}$. We have constructed $R$ in such a way that $\partial E \cap \partial R=\left\{z_{1}+s_{1} v, z_{2}+s_{2} v\right\}$ for some $s_{1}, s_{2} \in \mathbb{R}$.

Notice that $\bar{E} \cap \partial R$ is connected if and only if $\overline{X \backslash E} \cap \partial R$ is connected.

Assume by contradiction that $\bar{E} \cap \partial R$ is not connected, hence $\partial E \cap \partial R=\bar{E} \cap \partial R$ or $\partial E \cap \partial R=\overline{X \backslash E} \cap \partial R$. If $\partial E \cap \partial R=\bar{E} \cap \partial R$, let $F:=E \backslash R$, then $E \triangle F \Subset B_{\rho}$. Therefore, since $E \in \mathcal{M}_{\omega}\left(B_{\rho}\right)$, it follows

$$
P(E, R)=P\left(E, B_{\rho}\right)-P\left(F, B_{\rho}\right) \leq \omega(\rho) P\left(E, B_{\rho}\right) \leq \Theta \omega(\rho) \rho .
$$


On the other hand, there exists $c>0$ such that

$$
\begin{aligned}
P(E, R) & \geq c \mathcal{H}^{1}(\partial E \cap R) \geq c \mathcal{H}^{1}\left(\Gamma_{f} \cap R\right)-c \varepsilon \rho \\
& \geq c\left(\frac{c \eta}{K}-\varepsilon \rho\right) \geq \varepsilon \rho
\end{aligned}
$$

(for $\varepsilon$ sufficiently small) which gives a contradiction if we choose $\rho_{0}$ such that $\omega\left(\rho_{0}\right)<\varepsilon / \Theta$.

One can reason in a similar way if $\partial E \cap \partial R=\overline{X \backslash E} \cap \partial R$, by considering the set $F:=E \cup R$.

Lemma 6.2 (decay). There exist positive constants $\alpha, \beta, \gamma$ with $\beta<1 / 2$, which depend only on $(X,\|\cdot\|)$, such that if $E \in \mathcal{M}_{\omega}(\Omega), x_{0} \in \partial E, 0<\rho<\rho_{0}\left(2 L_{0}\right), \omega(\rho) \leq 1$ and $\mathbf{E c c}_{E}\left(x_{0}, 9 \rho\right) \leq \alpha$, then $\operatorname{Ecc}_{E}\left(x_{0}, 9 \beta \rho\right) \leq \gamma \omega(\rho)$.

Proof. Suppose for simplicity $x_{0}=0$. Let $v \in X,\|v\|=1$ be a vector such that $\mathbf{E c c}_{E}^{v}(0,9 \rho) \leq \alpha$ and let $\xi \in v^{*}$, $T:=\xi^{\perp}$. By Proposition 4.6, there exists a $2 L_{0}$-lipschitz function $f: T \rightarrow \mathbb{R}$ such that, letting $\rho^{\prime}:=\rho /\left(1+2 L_{0}\right)$, we have

$$
\mathcal{H}^{1}\left(\left(\partial E \triangle \Gamma_{f}\right) \cap B_{\rho^{\prime}}\right) \leq \alpha c\left(2 L_{0}\right) \rho^{\prime}
$$

If $\alpha c\left(2 L_{0}\right) \leq \varepsilon\left(2 L_{0}\right)$, by Lemma 6.1 it is possible to find a convex open set $R$, with $B_{\rho^{\prime} /\left(6+8 L_{0}\right)} \subset R \subset B_{\rho^{\prime}}$ and an half-space $H$ such that $\bar{H} \cap \partial R=\bar{E} \cap \partial R$ and $\partial H \cap \partial R=\partial E \cap \partial R$.

By Lemma 3.9 and Proposition 3.10 we have (noticing also that $\left\|\bar{D} \chi_{H}\right\|(R)=\left\|\bar{D} \chi_{H}(R)\right\|$, since $H$ is an half-space)

$$
\begin{aligned}
\left\|\bar{D} \chi_{E}\right\|(R) & =P(E, R) \leq\left(1+\omega\left(\rho^{\prime}\right)\right) P(H, R)+\omega\left(\rho^{\prime}\right) P\left(E, B_{\rho^{\prime}} \backslash R\right) \\
& =\left(1+\omega\left(\rho^{\prime}\right)\right)\left\|\bar{D} \chi_{H}(R)\right\|+\omega\left(\rho^{\prime}\right)\left\|\bar{D} \chi_{E}\right\|\left(B_{\rho^{\prime}} \backslash \Omega\right) \\
& =\left(1+\omega\left(\rho^{\prime}\right)\right)\left\|\bar{D} \chi_{E}(R)\right\|+\omega\left(\rho^{\prime}\right)\left\|\bar{D} \chi_{E}\right\|\left(B_{\rho^{\prime}} \backslash \Omega\right) \\
& \leq\left\|\bar{D} \chi_{E}(R)\right\|+\omega\left(\rho^{\prime}\right)\left\|\bar{D} \chi_{E}\right\|\left(B_{\rho^{\prime}}\right) .
\end{aligned}
$$

So that, by Proposition 3.2,

$$
\operatorname{Ecc}_{E}\left(0, \rho^{\prime} /\left(6+8 L_{0}\right)\right) \leq \frac{\left\|-D \chi_{E}\right\|(\Omega)-\left\|\bar{D} \chi_{E}(\Omega)\right\|}{\rho^{\prime} /\left(6+8 L_{0}\right)} \leq \Theta\left(6+8 L_{0}\right) \omega(\rho),
$$

and the proof is completed letting $\gamma:=\Theta\left(6+8 L_{0}\right), \beta:=\left(9\left(1+2 L_{0}\right)\left(6+8 L_{0}\right)\right)^{-1}$.

Proposition 6.3 (iteration). Let $\alpha, \beta, \gamma$ be the constants defined in Lemma 6.2 and let $\left.\rho_{1} \in\right] 0, \rho_{0}\left(2 L_{0}\right)\left[, \omega\left(\rho_{1}\right)\right.$ $\leq 1$, be such that $\omega\left(\rho_{1}\right) / \gamma<\alpha$. Then, for any $\left.\left.E \in \mathcal{M}_{\omega}(\Omega), x \in \partial E, \rho \in\right] 0, \rho_{1}\right]$, if $\operatorname{Ecc}_{E}(x, 2 \rho) \leq \alpha / 2$ then $\operatorname{Ecc}_{E}(y, \eta) \leq 2 \gamma \omega\left(\eta / \beta^{2}\right)$ for all $y \in B_{\rho}(x)$ and all $\left.\left.\eta \in\right] 0, \beta \rho\right]$.

In particular, if $\operatorname{Ecc}_{E}(x, 2 \rho) \leq \alpha / 2$ and $\left.\rho \in\right] 0, \rho_{1}\left[\right.$ then $\lim _{\eta \rightarrow 0^{+}} \operatorname{Ecc}_{E}(x, \eta)=0$.

Proof. By Proposition 4.3, for all $y \in B_{\rho}(x)$ we have $\operatorname{Ecc}_{E}(y, \rho) \leq 2 \mathbf{E c c}_{E}(x, 2 \rho) \leq \alpha$. So by Lemma 6.2 we find that $\operatorname{Ecc}_{E}(y, \beta \rho) \leq \gamma \omega(\rho)$ and by iteration we may conclude that for all positive integers $k$ there holds $\mathbf{E c c}_{E}\left(y, \beta^{k} \rho\right) \leq \gamma \omega\left(\beta^{k-1} \rho\right)$. Now, for any $\left.\left.\eta \in\right] 0, \beta \rho\right]$ we can find a positive integer $k$ such that $\beta^{k+1} \rho$ $\leq \eta \leq \beta^{k} \rho \leq \beta^{k-1} \rho \leq \eta / \beta^{2}$. Again, by Proposition 4.3 we get $\operatorname{Ecc}_{E}(y, \eta) \leq 2 \operatorname{Ecc}_{E}\left(y, \beta^{k} \rho\right) \leq 2 \gamma \omega\left(\beta^{k-1} \rho\right)$ $\leq 2 \gamma \omega\left(\eta / \beta^{2}\right)$.

By Proposition 6.3 we get

$$
\Omega \cap \Sigma(E)=\left\{x \in \Omega \cap \partial E: \operatorname{Ecc}_{E}(x, 2 \rho) \geq \alpha / 2 \quad \text { whenever } B_{\rho}(x) \subset \Omega, \rho \in\right] 0, \rho_{1}[\}
$$

and, in particular, $\Sigma(E)$ is relatively closed in $\Omega$ whenever $E \in \mathcal{M}_{\omega}(\Omega)$. 


\subsection{The uniformly convex case}

The following regularity result for a set $E \in \mathcal{M}_{\omega}(\Omega)$ in the uniformly convex case easily follows from Proposition 5.1, Proposition 6.3 and the definition of $\Sigma(E)$.

Theorem 6.4. Let $E \in \mathcal{M}_{\omega}(\Omega)$ and $x_{0} \in \partial E \backslash \Sigma(E)$. Assume also that $\omega(\rho) \leq C \rho^{\alpha}$, for some constants $C, \alpha>0$, and that $B_{1}^{*}$ is uniformly convex. Then, there exists a neighbourhood $U$ of $x_{0}$ such that $U \cap \partial E$ is a $\mathcal{C}^{1, \alpha / 2}$ curve.

\subsection{The convex case: Minimal sets}

We can now prove that minimal sets are locally lipschitz graphs out of the singular set, even without uniform convexity assumptions on $\partial B_{1}^{*}$. Indeed, from Lemma 6.2 and Proposition 4.3 we obtain the following result (see also [24], Th. 4.3).

Proposition 6.5 (regularity). Let $E \in \mathcal{M}_{0}(\Omega), x_{0} \in \partial E \backslash \Sigma(E)$. Then, for all $L>L_{0}$ there exists $\rho>0$ such that $\partial E \cap B_{\rho}\left(x_{0}\right)$ is the graph of a L-lipschitz function.

\subsection{The convex case: $\boldsymbol{\omega}$-minimal sets}

In this section we consider general $\omega$-minimal sets, assuming for the sake of simplicity that $\Omega=X$.

We will prove in Theorem 6.14 that these sets are locally parameterizable by means of a bi-lipschitz map out of the singular set. Moreover, it is not true in the general case that a set $E \in \mathcal{M}_{\omega}(\Omega)$ is locally the graph of a lipschitz function, as it happens in the case of minimal sets (see Sect. 7 for a counter-example).

Notice that since for a $\omega$-minimal set $E$ we have $\mathcal{H}^{n-1}(\partial E)=\mathcal{H}^{n-1}\left(\partial^{*} E\right)<+\infty$ we get $|\partial E|=0$. This fact ensures that $|\bar{E} \backslash \stackrel{\circ}{E}|=0$ that is $E, \bar{E}$ and $\stackrel{\circ}{E}$ are all equivalent in measure. Moreover notice that, in this case, $\partial E$ is the usual topological boundary of $\stackrel{\circ}{E}$. In the following we will often consider the connected components of $\stackrel{\circ}{E}$ and, as well, the connected components of $X \backslash \bar{E}$. The following result linking topological to measure theoretic decomposition will be used implicitly (see [7], Th. 2).

Theorem 6.6. Let $E \subset X$ be an open set with finite perimeter and let $\left\{E_{i}\right\}_{i \in I}$ be the family of the connected components of $E$. Suppose moreover $\mathcal{H}^{n-1}\left(\partial E \backslash \partial^{*} E\right)=0$. Then $I$ is at most countable and, for all open sets $A \subset \mathbb{R}^{n}$,

$$
P(E, A)=\sum_{i \in I} P\left(E_{i}, A\right) .
$$

We are interested in connected components for the following regularity result (see [7], Th. 7).

Theorem 6.7 (Jordan). Let $E \subset \mathbb{R}^{2}$ be bounded, and simply connected (in the sense that both $\stackrel{\circ}{E}$ and $\mathbb{R}^{2} \backslash \bar{E}$ are connected) and with finite perimeter. Then $\partial E$ is a closed Jordan curve, i.e. there exists a lipschitz continuous curve $\gamma:[0,1] \rightarrow \partial E$ such that $\gamma(0)=\gamma(1)$ and $\gamma_{\mid[0,1[}$ is injective.

Here is the idea which motivates the following lemmata. First we prove that $E$ is "locally simply connected". This is achieved by noticing that large connected components cannot be too close, in fact we already know that $\partial E$ is "almost" the graph of a function. On the other hand, small connected components cannot exists due to the minimality of $E$. Once we have stated that $\partial E$ is locally the boundary of a simply connected set we can apply Jordan's theorem to get a local lipschitz parameterization of the boundary. Finally we get estimates on the Lipschitz constant from the $\omega$-minimality of $\partial E$ with respect to straight lines.

In the following $E$ will be an $\omega$-minimal set of $X(\operatorname{dim} X=2)$.

Lemma 6.8. There exists a constant $c>0$ such that $P(E) \geq c\|x-y\|$ for all $x, y \in \partial E$, whenever $\stackrel{\circ}{E}$ is connected and bounded. 
Proof. Suppose for simplicity $x=0$ and let $T$ be the line through $x$ and $y$. Let $\xi \in X^{*}$ be such that $\|\xi\|=1$ and $\xi^{\perp}=T$ and let $v \in \xi^{*}$. Consider the projection $\pi: X \rightarrow T$ defined by $\pi(z+t v)=z$ for all $z \in T, t \in \mathbb{R}$. Then $\pi(\partial E) \supset[x, y]$ otherwise if there were $z \in[x, y] \backslash \pi(\partial E)$ the line $\pi^{-1}(z)$ (which cannot be all contained in $\stackrel{\circ}{E}$, since $\stackrel{\circ}{E}$ is bounded) would decompose $\stackrel{\circ}{E}$ in two parts.

So there exist positive constants $c_{1}, c_{2}, c_{3}$ such that

$$
P(E) \geq c_{1} \mathcal{H}^{1}(\partial E) \geq c_{2} \mathcal{H}^{1}(\pi(\partial E)) \geq c_{2} \mathcal{H}^{1}([x, y]) \geq c_{3}\|x-y\| .
$$

Lemma 6.9. Given an orientation on $X$, there exists a convex and positively 1 -homogeneous function $\psi: X \rightarrow \mathbb{R}$ such that, given any closed lipschitz Jordan curve $\gamma:[0, L] \rightarrow X$ inducing the given orientation on $X$, we have

$$
P(E, X)=\int_{0}^{L} \psi\left(\gamma^{\prime}(t)\right) \mathrm{d} t
$$

where $E$ is the set bounded by $\gamma$.

Proof. Given an orientation on $X$, we can construct a continuous bijection $A: X \rightarrow X^{*}$ in the following way. For a given $v \in X$ we consider the straight line spanned by $v$ (which is an hyper-space $\operatorname{since} \operatorname{dim} X=2$ ). The direction of $v$ together with the orientation of $X$ enable us to choose one of the two half-spaces determined by the straight line. This half-space has a constant normal vector $\nu$. So we let $A v=\|v\|_{s} \nu$.

From Theorem 2.7 we get

$$
P(E, X)=\int_{\partial E} \varphi\left(\nu_{E}(x)\right) \mathrm{d} \mathcal{H}^{1}(x)=\int_{0}^{L} \varphi\left(\nu_{E}(\gamma(t))\right)\left\|\gamma^{\prime}(t)\right\|_{s} \mathrm{~d} t=\int_{0}^{L} \psi\left(\gamma^{\prime}(t)\right) \mathrm{d} t,
$$

where we have set $\psi(v)=\varphi(A v)$.

Corollary 6.10. Let $\lambda: X \rightarrow \mathbb{R}^{2}, \varphi^{\circ}: \mathbb{R}^{2} \rightarrow \mathbb{R}$ be as in Section 2.5. Given an orientation on $X$ and on $\mathbb{R}^{2}$, assume that $\lambda$ preserves the orientations. Let also $\psi: X \rightarrow \mathbb{R}$ be as in Lemma 6.9. Then we have

$$
\psi(v)=\varphi^{o}(R \lambda v), \quad \forall v \in X,
$$

where $R: \mathbb{R}^{2} \rightarrow \mathbb{R}^{2}$ is the $\frac{\pi}{2}$ clock-wise rotation of $\mathbb{R}^{2}$ inducing the given orientation.

Proof. In fact, let $H=H_{\nu}$ be the half-space such that $A v=\|v\|_{s} \nu$ that is $\lambda H$ is the half-space on the left of $\lambda v$. Notice that $R \lambda v=|\lambda v| \nu_{\lambda H}$. So, if we choose an open set $B$ such that $B \cap \partial H=[0, v]$, by the representation formula in Section 2.5 we get

$$
P(H, B)=\int_{\partial H} \varphi^{o}\left(\nu_{\lambda H}(x)\right) \mathrm{d} \mathcal{H}^{1}(x)=\frac{\varphi^{o}(R \lambda v)}{|\lambda v|} \mathcal{H}^{1}(\lambda[0, v])=\varphi^{o}(R \lambda v) .
$$

While, letting $\gamma(t)=v t$, by the representation formula in the previous lemma, we get

$$
P(H, B)=\int_{0}^{1} \psi\left(\gamma^{\prime}(t)\right) \mathrm{d} t=\psi(v) .
$$

For a set $C \subset X$, we let $\operatorname{rad}(C)$ be the radius of $C$ that is

$$
\operatorname{rad}(C)=\inf _{x \in C} \sup _{y \in C}\|y-x\| .
$$

Notice that $\operatorname{diam}(C) \leq 2 \operatorname{rad}(C)$ and that, in general, the equality does not hold. 
Lemma 6.11. There exists a constant $c \in] 0,1\left[\right.$ such that, given $E \in \mathcal{M}_{\omega}$, if $E_{0}$ is a connected component of $\stackrel{\circ}{E}$ or of $X \backslash \bar{E}$ then $\omega\left(2 \operatorname{rad}\left(\partial E_{0}\right)_{+}\right) \geq c$.

Proof. Let $B$ be a ball of radius $\rho>2 \operatorname{rad}\left(\partial E_{0}\right)$ centered on $\partial E_{0}$ and such that $\partial E_{0} \Subset B$. We may also suppose that $E_{0}$ is a connected component of $\stackrel{\circ}{E}$ and $E_{0} \Subset B$; otherwise we could consider the set $X \backslash E$ instead of $E$. Let $F:=E \backslash E_{0}$, so that $E \triangle F \Subset B$. By the minimality of $E$ :

$$
P(E, B) \leq(1+\omega(\rho)) P(F, B) .
$$

Since $E_{0}$ is a connected component of $\stackrel{\circ}{E}$ we also have $P(E, B)=P(F, B)+P\left(E_{0}, B\right)$ so that we conclude $P\left(E_{0}, X\right)=P\left(E_{0}, B\right) \leq \omega(\rho) P(E, B) \leq \Theta \rho \omega(\rho)$. On the other hand, by Lemma 6.8 we get also $P\left(E_{0}, B\right)$ $\geq C \operatorname{rad}\left(\partial E_{0}\right)=(C / 2) \rho$, and the conclusion follows.

Proposition 6.12. If $E \in \mathcal{M}_{\omega}, R \subset X$ is an open, bounded and convex set such that $\bar{E} \cap \partial R$ is connected, $\partial E \cap \partial R$ contains exactly two points and $\omega(2 \operatorname{rad} R)<c$, with $c$ as in Lemma 6.11. Then $\stackrel{E^{\circ}}{R \cap}$ and $X \backslash \overline{E \cap R}$ are both connected.

Proof. Notice that $\partial R$ is homeomorphic to a circle, and $\partial R=I_{1} \cup I_{2} \cup\left\{x_{1}, x_{2}\right\}$ where $I_{1}$ and $I_{2}$ are disjoint, connected and relatively open sets, $\left\{x_{1}, x_{2}\right\}=\partial E \cap \partial R, I_{1} \subset \stackrel{\circ}{E}$ and $I_{2} \subset X \backslash \bar{E}$.

Let $E_{0}$ be a connected component of $\stackrel{\circ}{E} \cap R$ and let $E_{0}^{\prime}$ be the connected component of $\stackrel{\circ}{E}$ containing $E_{0}$. First suppose that $E_{0}=E_{0}^{\prime}$. In this case, being $E_{0}$ a connected component of $\stackrel{\circ}{E}$, the hypothesis $\omega(2 \operatorname{rad} \Omega)<c$ is in contradiction with Lemma 6.11. On the other hand, if $E_{0} \neq E_{0}^{\prime}$, we conclude that $I_{1} \cap E_{0}^{\prime}$ is not empty. This means that all the connected components of $\stackrel{\circ}{E}$ that meet $R$ also meet $I_{1}$. Since $I_{1}$ is connected, we conclude that $\stackrel{\circ}{E} \cap R$ has a single connected component.

Reasoning the same way with the set $X \backslash E$ we conclude the proof.

Lemma 6.13. Let $R>0, E \in \mathcal{M}_{\omega}\left(B_{3 R}\left(z_{0}\right)\right), z_{0} \in \partial E$ and $\Omega \subset B_{R}\left(z_{0}\right)$ an open, convex set, such that $\partial E \cap \bar{\Omega}$ is parameterizable by means of a injective, lipschitz curve $\gamma:[0, L] \rightarrow \bar{\Omega}$ with $\gamma^{-1}(\partial \Omega)=\{0, L\}$. Then, there exist positive constants $C, \varepsilon$ such that if $\omega(2 R) \leq C$ then for any couple of points $x_{0}=\gamma\left(t_{0}\right), x_{1}=\gamma\left(t_{1}\right)$, with $\left.t_{0}, t_{1} \in\right] 0, L[$, we have

$$
\int_{t_{0}}^{t_{1}} \psi\left(\gamma^{\prime}(t)\right) \mathrm{d} t \leq C\left\|x_{1}-x_{0}\right\|
$$

Proof. Suppose for simplicity $t_{0} \leq t_{1}$. Suppose also $\psi\left(\gamma^{\prime}(t)\right)=1$ for a.e. $t \in[0, L]$ so that $P(E, \Omega)=L$. Let $s_{0}:=\min \left\{t \in[0, L]: \gamma(t) \in\left[x_{0}, x_{1}\right]\right\}$ and $s_{1}:=\max \left\{t \in[0, L]: \gamma(t) \in\left[x_{0}, x_{1}\right]\right\}$. Clearly we have $0 \leq s_{0} \leq t_{0}$ $\leq t_{1} \leq s_{1} \leq L$ and we may define the curve $\sigma:[0, L] \rightarrow \Omega$ by

$$
\sigma(t):= \begin{cases}\gamma(t) & \text { if } t \in\left[0, s_{0}\right] \cup\left[s_{1}, L\right], \\ \frac{s_{1}-t}{s_{1}-s_{0}} \gamma\left(s_{0}\right)+\frac{t-s_{0}}{s_{1}-s_{0}} \gamma\left(s_{1}\right) & \text { if } \quad t \in] s_{0}, s_{1}[.\end{cases}
$$

Note that $\sigma$ is injective and $\sigma^{-1}(\partial \Omega)=\gamma^{-1}(\partial \Omega)=\{0, L\}$, therefore the set $\bar{\Omega} \backslash \sigma([0, L])$ has exactly two connected components. If we call $G$ the connected component such that $\bar{G} \cap \partial \Omega=\bar{E} \cap \partial \Omega$ and let $F:=(E \backslash \Omega) \cup G$ we have $F \triangle E \Subset \Omega$. Moreover, we find that

$$
\begin{aligned}
P(F, \Omega)-P(E, \Omega) & =\psi\left(\gamma\left(s_{1}\right)-\gamma\left(s_{0}\right)\right)-\int_{s_{0}}^{s_{1}} \psi\left(\gamma^{\prime}(t)\right) \mathrm{d} t \\
& \leq k \psi\left(\gamma\left(t_{1}\right)-\gamma\left(t_{0}\right)\right)-\int_{s_{0}}^{s_{1}} \psi\left(\gamma^{\prime}(t)\right) \mathrm{d} t
\end{aligned}
$$

where $k$ is such that $\max \{\psi(v), \psi(-v)\} \leq k \psi(v)$, for any $v \in X$. 
Set now $l_{1}:=t_{1}-t_{0}, l_{2}:=\left(s_{1}-s_{0}\right)-\left(t_{1}-t_{0}\right)$ and $s:=\psi\left(x_{1}-x_{0}\right)$. Let also $r>0$ be such that $E \triangle F \Subset B_{2 r}\left(x_{0}\right)$. Note that

$$
P(F, \Omega)-P(E, \Omega)=P\left(F, B_{2 r}\left(x_{0}\right)\right)-P\left(E, B_{2 r}\left(x_{0}\right)\right)
$$

hence by the minimality property of $E$ and by Proposition 3.2 we obtain

$$
P(F, \Omega)-P(E, \Omega) \geq-\frac{2 \Theta \omega(2 r)}{1+\omega(2 r)} r \geq-\frac{2 \Theta \omega(2 R)}{1+\omega(2 R)} r
$$

If $r \leq s$, from (11) and (12) we get

$$
k s-l_{1} \geq k s-l_{1}-l_{2} \geq-\frac{2 \Theta \omega(2 R)}{1+\omega(2 R)} s,
$$

whence

$$
l_{1} \leq\left(k+\frac{2 \Theta \omega(2 R)}{1+\omega(2 R)}\right) s
$$

If $r>s$ we can assume, possibly reducing $r$, that at least one of the curves $\gamma\left(\left[t_{0}, t_{1}\right]\right), \gamma\left(\left[s_{0}, t_{0}\right] \cup\left[t_{1}, s_{1}\right]\right)$ intersects $B_{2 r}\left(x_{0}\right) \backslash B_{r}\left(x_{0}\right)$, hence either $l_{1} \geq c(r-s)$ or $l_{2} \geq c(r-s)$ for some constant $c>0$, depending only on $(X,\|\cdot\|)$. If $R$ is such that $\frac{2 \Theta \omega(2 R)}{1+\omega(2 R)} \leq \frac{c}{2}$, from (11) and (12) we get

$$
k s-l_{1}-l_{2} \geq-\frac{2 \Theta \omega(2 R)}{1+\omega(2 R)} r \geq-\frac{c}{2} r
$$

whence,

$$
k s-\frac{l_{1}}{2} \geq \frac{l_{1}}{2}+l_{2}-\frac{c}{2} r \geq-\frac{c}{2} s .
$$

So, we finally obtain

$$
l_{1} \leq 2\left(k+\frac{c}{2}\right) s
$$

which concludes the proof.

Using Lemma 6.13, we can prove the following regularity result:

Theorem 6.14. Let $E \in \mathcal{M}_{\omega}, x_{0} \in \partial E \backslash \Sigma(E)$. Then, there exists a neighbourhood of $x_{0}$ where $\partial E$ is parameterizable by means of a bi-lipschitz map.

Proof. By Lemma 6.1 we may find a convex open neighbourhood $\Omega$ of $x$ such that $\partial E \cap \partial \Omega$ consists of exactly two points. By Proposition 6.12 we may also suppose that $\stackrel{\circ}{E} \cap \Omega$ is connected so that, by Theorem 6.7 , we get that $\partial E \cap \bar{\Omega}$ is parameterizable by means of a bijective lipschitz curve $\gamma:[0, L] \rightarrow \partial E \cap \bar{\Omega}$. Finally Lemma 6.13 proves that $\gamma^{-1}$ is also lipschitz.

Note also that in the previous theorem it is possible to give uniform bounds on the radius of the neighbourhood of $x$ in which there is a bi-lipschitz parameterization, and on the bi-lipschitz constant. 


\subsection{Everywhere regularity}

We now want to study the structure of the singular set $\Sigma(E)$, for $E \in \mathcal{M}_{\omega}(\Omega)$. We prove that in most cases (depending on the shape of $\left.B_{1}\right)$ the set $\Sigma(E)$ is empty (Th. 6.18).

We recall this general property of closed convex subsets of $X$.

Lemma 6.15. Let $C \subset X$ be closed and convex. For $k \geq 2$, let $x_{1}, \ldots, x_{k}$ be extremal points of $C$ with $x_{i} \neq x_{j}$ for $i \neq j$. Let also $0 \leq \alpha_{i} \leq \pi$ be the angle defined by $C$ in $x_{i}$. Then there holds

$$
\sum_{i=1}^{k} \alpha_{i} \geq(k-2) \pi
$$

and the equality holds if and only if $C$ is the " $k$-agon" (possibly degenerate) spanned by $x_{1}, \ldots, x_{k}$.

Proof. Notice that the $k$-agon spanned by $x_{1}, \ldots, x_{k}$ has angles $\beta_{i} \leq \alpha_{i}$ such that $\sum_{i} \beta_{i}=(k-2) \pi$. This proves the first statement. To prove the second statement notice that a convex set is contained in each of its angles so that if $\alpha_{i}=\beta_{i}$ for all $i$, then the convex set is contained in the polyhedron.

In the following proposition we point out some properties of the two-dimensional minimal cones (see also [29], Ref. [24], Th. 3.2).

Proposition 6.16. Let $E \in \mathcal{M}_{0}(X)$ be a cone over the origin, then one of the following conditions hold:

1. $\operatorname{Ecc}_{E}(0, R)=0$ for any $R>0$ and $\partial E$ is the union of two half-lines starting from the origin;

2. $B_{1}$ is a quadrilateral and $\partial E$ is the union of four half-lines;

3. $B_{1}$ is a triangle and $\partial E$ is the union of four or six half-lines.

Proof. Since $E \in \mathcal{M}_{0}(X), \partial E$ is the union of a finite number (even) of half-lines starting from the origin, say $l_{1}, \ldots, l_{N}$ (we label the half-lines such that $l_{i}$ and $l_{i+1}$ are consecutive). For any $i \in\{1, \ldots, N\}$, we can find an extremal point $x_{i} \in \partial B_{1}$ such that, denoting by $C_{i}$ the cone bounded by $l_{i}$ and $l_{i+1}$, if $C_{i}$ is contained in $E$ then $C_{i}$ contains the set $B_{1}-x_{i}$, whereas if $C_{i}$ is contained in $X \backslash E$ then it contains the set $x_{i}-B_{1}$. Indeed, assuming that $C_{i}$ is contained in $E$ and defines an angle smaller than $\pi$, we can consider the set $E \backslash T$, where $T$ is a triangle having a vertex in the origin and two edges contained in $l_{i}$ and $l_{i+1}$. Then, the assertion follows from the minimality property of $E$ with respect to $E \backslash T$. More precisely, from Jensen's inequality it follows that the set $C_{i} \backslash T$ has perimeter smaller than or equal to the perimeter of $C_{i}$, and the equality holds if and only if the exterior normals to $C_{i} \backslash T$ lie all in the same edge of $B_{1}^{*}$, which is equivalent to say that $C_{i}$ contains the set $B_{1}-x_{i}$. One can proceed in a similar way when the angle between $l_{i}$ and $l_{i+1}$ is contained in $X \backslash E$. If $N=2$, this implies $\operatorname{Ecc}_{E}(0, R)=0$ for any $R>0$ as $\nu_{E}(x) \in x_{1}^{*}$ for all $x \in \partial E \backslash\{0\}$. Let us consider the case $N \geq 4$. Assume that $x_{i}=x_{j}$ for some $i \neq j$. Observe that the same point $x_{i}$ can occur at most two times, in correspondence of an angle contained in $E$ and of an angle contained in $X \backslash E$. Since $N>2$, possibly changing the first half-line $l_{1}$ of the enumeration and the orientation, we can assume $i=1$ and $j \geq 4$. Let us consider the points $x_{1}, x_{2}$ and $x_{3}$; they are all distinct and, if $\alpha_{k}$ is the angle defined by $B_{1}$ in $x_{k}$, there holds $\alpha_{1}+\alpha_{2}+\alpha_{3} \leq \pi$ (see Fig. 1). By Lemma 6.15, this implies that $B_{1}$ is the triangle spanned by $x_{1}, x_{2}, x_{3}$, and $N \in\{4,6\}$ (see Fig. 2). It follows that, if $B_{1}$ is not a triangle, the map $i \rightarrow x_{i}$ is an injection. Let $\alpha_{i}$ be the angle defined by $B_{1}$ in $x_{i}$. We have

on the other hand, from Lemma 6.15 it follows

$$
\sum_{i=1}^{N} \alpha_{i} \leq 2 \pi
$$

$$
\sum_{i=1}^{N} \alpha_{i} \geq(N-2) \pi
$$

which implies $N=4$ and so $B_{1}$ coincides with the polygon spanned by $x_{1}, x_{2}, x_{3}, x_{4}$ (see Fig. 2). 


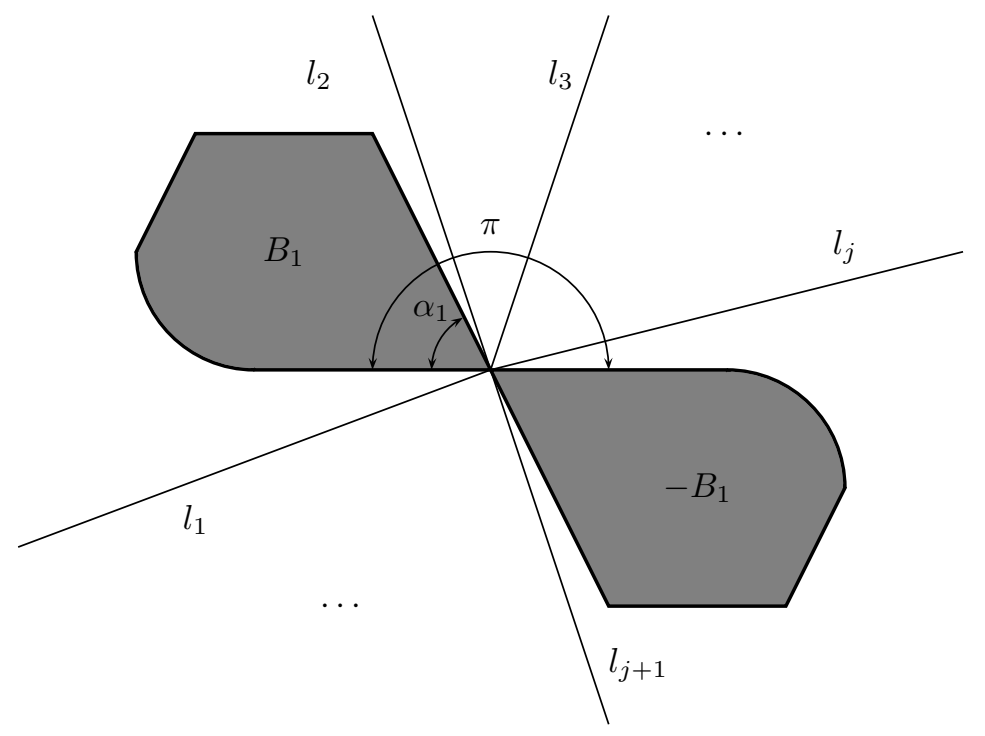

Figure 1. The construction in the proof of Proposition 6.16.
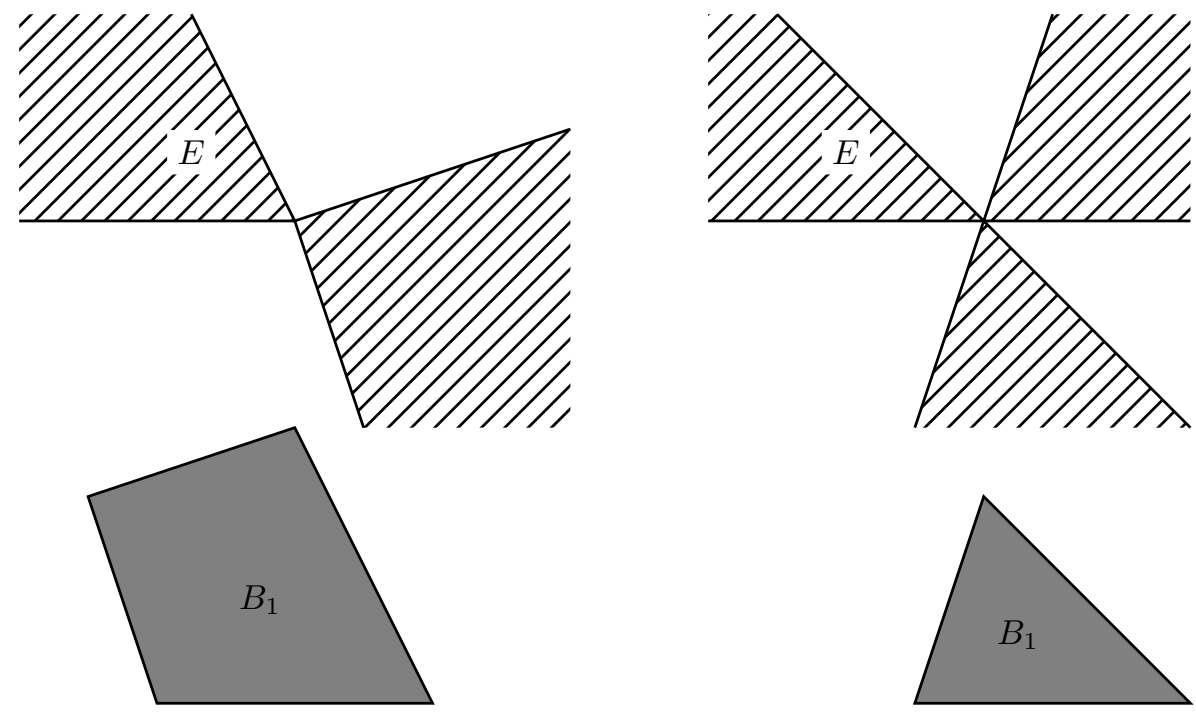

Figure 2. The singular minimal cones.

Theorem 6.17. Let $E \in \mathcal{M}_{0}(\Omega)$ and $x_{0} \in \partial E$. Then, there exist $\rho>\eta>0$ and a set $C$ with $C \triangle E \Subset B_{\rho}\left(x_{0}\right)$ such that $C \cap B_{\eta}\left(x_{0}\right)$ is a cone on $x_{0}$ and $P\left(C, B_{\rho}\left(x_{0}\right)\right)=P\left(E, B_{\rho}\left(x_{0}\right)\right)$. Moreover, if $x_{0} \in \Sigma(E)$ then $B_{1}$ is either a triangle or a quadrilateral.

Proof. Set for simplicity $x_{0}=0$ and fix $\rho>0$ such that $B_{\rho} \subset \Omega$. Let $E_{k}, k \in I$, be the connected components of $\stackrel{\circ}{E} \cap B_{\rho}$ and let $E_{k}^{\prime}, k \in I^{\prime}$, be the connected components of $B_{\rho} \backslash \bar{E}$ ( $I$ and $I^{\prime}$ are at most countable). Since $E$ is minimal, $P\left(E_{k}, \partial B_{\rho}\right)>0$ and $P\left(E_{k}^{\prime}, \partial B_{\rho}\right)>0$, otherwise we could consider $E \backslash E_{k}$ or $E \cup E_{k}^{\prime}$ and reduce the perimeter of $E$. Moreover, the set $J=\left\{k \in I: 0 \in \partial E_{k}\right\}$ is finite, otherwise by Lemma 6.8 we had $P\left(E, B_{\rho}\right)=+\infty$. The same is true for the set $J^{\prime}=\left\{k \in I^{\prime}: 0 \in \partial E_{k}^{\prime}\right\}$. Let us fix a radius $\eta<\rho$ such that if $0 \notin \partial E_{k}$ then $\partial E_{k} \cap B_{\eta}=\emptyset$ for any $k \in J$, and such that the same is true for the sets $E_{k}^{\prime}$ for any $k^{\prime} \in J^{\prime}$. Clearly, 
we can choose a strictly positive $\eta$, otherwise we had infinitely many components $E_{k}$ (or $E_{k}^{\prime}$ ) intersecting the ball $B_{\rho / 2}$, which would imply again $P\left(E, B_{\rho}\right)=+\infty$.

We focus on a given component $E_{k}$ of $\stackrel{\circ}{E} \cap B_{\rho}$ with $k \in J$. Notice that $E_{k}$ is simply connected and $\partial E_{k}$ is a closed Jordan curve passing through 0 . Notice also that all the connected components of $\partial E_{k} \cap B_{\rho}$ which intersect $B_{\eta}$ contain 0 otherwise there should be a component $E_{j}^{\prime}$ with $j \in J$ which intersects $B_{\eta}$ but such that $0 \notin \partial E_{j}^{\prime}$. So only one connected component of $\partial E_{k} \cap B_{\rho}$ intersects $B_{\eta}$. Moreover, $\partial E_{k} \cap \partial E_{j}=\{0\}$ for any $k, j \in J, k \neq j$. Indeed, if $\left(\partial E_{k} \cap \partial E_{j}\right) \backslash\{0\} \neq \emptyset$, there would exist a component $E_{h}^{\prime}$ of $B_{\rho} \backslash \bar{E}$ such that $P\left(E_{h}^{\prime}, \partial B_{\rho}\right)=0$, which contradicts the minimality of $E$ as above.

Let us take now a lipschitz parameterization of $\partial E_{k}$ given by $\gamma_{k}:[0,3] \rightarrow \overline{B_{\rho}}$ such that $\gamma_{k}(0)=\gamma_{k}(3)=0$, $\gamma_{k}(1) \in \partial B_{\eta}, \gamma_{k}(2) \in \partial B_{\eta}, \gamma_{k}(3 / 2) \in \partial B_{\rho}$ and $\gamma_{k}(t) \in B_{\rho} \backslash \overline{B_{\eta}}$ for any $\left.t \in\right] 1,2[$. Observe that the two points $\gamma_{k}(1), \gamma_{k}(2) \in \partial B_{\eta}$ are independent of the choice of the parameterization $\gamma_{k}$.

Then, we consider the lipschitz curve $\alpha_{k}:[0,3] \rightarrow \overline{B_{\rho}}$ defined as

$$
\alpha_{k}(t)= \begin{cases}t \gamma_{k}(1) & \text { if } t \in[0,1] \\ \gamma_{k}(t) & \text { if } t \in[1,2] \\ (3-t) \gamma_{k}(2) & \text { if } t \in[2,3]\end{cases}
$$

Notice that also $\alpha_{k}$ defines a closed Jordan curve, and let us denote by $C_{k}$ the compact subset of $X$ bounded by $\alpha_{k}$. By the definition of $\alpha_{k}$ it follows $E_{k} \triangle C_{k} \Subset B_{\rho}$, moreover $C_{k} \cap C_{j}=\{0\}$ for any $k, j \in J, k \neq j$. Indeed, $\left(C_{k} \cap C_{j}\right) \backslash\{0\} \neq \emptyset$ implies $\left(\partial E_{k} \cap \partial E_{j}\right) \backslash\{0\} \neq \emptyset$, which gives a contradiction.

From Lemma 6.9 and Jensen's inequality we obtain

$$
\begin{aligned}
P\left(C_{k}, \overline{B_{\rho}}\right) & =\int_{0}^{1} \psi\left(\alpha_{k}^{\prime}(t)\right) \mathrm{d} t+\int_{1}^{2} \psi\left(\alpha_{k}^{\prime}(t)\right) \mathrm{d} t+\int_{2}^{3} \psi\left(\alpha_{k}^{\prime}(t)\right) \mathrm{d} t \\
& \leq \int_{0}^{1} \psi\left(\gamma_{k}^{\prime}(t)\right) \mathrm{d} t+\int_{1}^{2} \psi\left(\gamma_{k}^{\prime}(t)\right) \mathrm{d} t+\int_{2}^{3} \psi\left(\gamma_{k}^{\prime}(t)\right) \mathrm{d} t \\
& =P\left(E_{k}, \overline{B_{\rho}}\right) .
\end{aligned}
$$

The first statement of the theorem follows by setting $C:=\left(E \backslash \bigcup_{k \in J} E_{k}\right) \cup \bigcup_{k \in J} C_{k}$.

Notice that, by the minimality of $E$, the equality holds in Jensen's inequality (14), therefore

$$
\begin{aligned}
\int_{0}^{1} \psi\left(\gamma_{k}^{\prime}(s)\right) \mathrm{d} s & =\int_{0}^{1} \psi\left(\alpha_{k}^{\prime}(s)\right) \mathrm{d} s=\psi\left(\gamma_{k}(1)\right)=\psi\left(\int_{0}^{1} \gamma_{k}^{\prime}(s) \mathrm{d} s\right) \\
\int_{2}^{3} \psi\left(\gamma_{k}^{\prime}(s)\right) \mathrm{d} s & =\int_{2}^{3} \psi\left(\alpha_{k}^{\prime}(s)\right) \mathrm{d} s=\psi\left(\gamma_{k}(2)\right)=\psi\left(\int_{2}^{3} \gamma_{k}^{\prime}(s) \mathrm{d} s\right) .
\end{aligned}
$$

This implies that $\psi$ is linear on the convex hull of $\left\{\gamma_{k}^{\prime}(s): s \in[0,1]\right\}$ and on the convex hull of $\left\{\gamma_{k}^{\prime}(s): s \in[2,3]\right\}$. This is equivalent to say that $\nu_{E_{k}}\left(\gamma_{k}(s)\right)$ belongs to the same edge $v_{k}^{*}$ of $B_{1}^{*}$ for a.e. $s \in[0,1]$ (and respectively to $w_{k}^{*} \subset \partial B_{1}^{*}$ for a.e. $\left.s \in[2,3]\right)$.

Suppose now $x_{0}=0 \in \Sigma(E)$. This implies $0 \in \Sigma(C)$, in fact if by contradiction $\mathbf{E c c}_{C}^{v}(0, \eta)=0$, it follows that $J=\{0\}$ and $v=v_{0}=w_{0}$, so that $\operatorname{Eccc}_{E}^{v}(0, \eta)=0$ and $0 \notin \Sigma(E)$.

Let $C_{\infty}$ be the blow-up of $C$ in 0 (note that $C_{\infty} \cap B_{\eta}=C \cap B_{\eta}$ ). Since $C_{\infty} \in \mathcal{M}_{0}(X)$ and $0 \in \Sigma\left(C_{\infty}\right)$, by Proposition 6.16 it follows that $B_{1}$ (hence $B_{1}^{*}$ ) is a triangle or a quadrilateral.

Theorem 6.18. Let $E \in \mathcal{M}_{\omega}(\Omega)$. If $B_{1}$ is neither a triangle nor a quadrilateral then $\Sigma(E)=\emptyset$.

Proof. Given any $x \in \partial E$ consider the sets $E_{k}=k(E-x)$. By Proposition 3.3, up to a subsequence, we may suppose that $E_{k} \rightarrow E_{\infty}$ with $E_{\infty} \in \mathcal{M}_{0}$. Since $0 \in \partial E_{k}$ we also have $0 \in \partial E_{\infty}$. Now note that $0 \notin \Sigma\left(E_{\infty}\right)$ otherwise by Theorem $6.17 E_{\infty}$ would be a singular minimal cone and this contradicts Proposition 6.16. So, there exist $\rho>0$ such that $\operatorname{Ecc}_{E_{\infty}}(0,2 \rho)<\alpha / 2$ and, by Lemma 6.2 , we can find a radius $\eta \leq \rho$ such 
that $\mathbf{E c c}_{E_{\infty}}(0, \eta)=0$. Possibly considering an even smaller $\eta$, by Proposition 4.3 we may suppose that $\mathbf{E c c}_{E_{\infty}}(0, \eta)=\lim _{k \rightarrow \infty} \mathbf{E c c c}_{E_{k}}(0, \eta)$. Therefore we obtain $\lim _{k \rightarrow \infty} \operatorname{Ecc}_{E}(x, \eta / k)=0$ and Lemma 6.2 again gives $x \notin \Sigma(E)$.

\subsection{Sets with prescribed curvature}

In this section we consider sets $E \in \mathcal{B}(X)$ that minimize the functional

$$
E \mapsto P(E, \Omega)+\int_{E \cap \Omega} H(x) \mathrm{d} x
$$

where $H \in L^{\infty}(\Omega)$ and $\Omega \subset X$ is an open set. It is easy to check that these sets are additive $\omega$-minimizers (see Remark 3.7) and in particular are $\omega$-minimizers. But in this case stronger regularity results hold.

Theorem 6.19 ( $C^{1,1}$ regularity). Assume that $B_{1}$ is not a triangle. Let $E \subset X$ be a minimal set for (16), and let $x_{0} \in(\partial E \cap \Omega) \backslash \Sigma(E)$. Then, there exists a neighbourhood $U$ of $x_{0}$ where $\partial E$ is the graph of a lipschitz function. Moreover, there exists a lipschitz function $v: \partial E \cap U \rightarrow \partial B_{1}$ such that $v(y) \in \nu_{E}(y)^{*}$ for any $y \in \partial^{*} E \cap U$.

Proof. From Theorem 6.14 we already know that there exists a convex neighbourhood $U \subseteq \Omega$ of $x_{0}$ such that $\partial E \cap U$ is parameterizable by means of a bi-lipschitz map $\gamma:] 0, L[\rightarrow U$. By Lemma 6.9, there exists a convex function $\psi$ such that

$$
P(E, U)=\int_{0}^{L} \psi\left(\gamma^{\prime}(t)\right) \mathrm{d} t
$$

Suppose also $\psi\left(\gamma^{\prime}(t)\right)=1$ for a.e. $\left.t \in\right] 0, L[$, so that $L=P(E, U)$.

Fix now Lebesgue points $\left.t_{1}, t_{2} \in\right] 0, L\left[\right.$ and let $a:=\gamma\left(t_{1}\right), b:=\gamma\left(t_{2}\right)$. Choose also $0<\delta<\left(t_{2}-t_{1}\right) / 2, v \in B_{1}$ and consider the function $\varphi_{\delta}$ defined by

$$
\varphi_{\delta}(t):=\left\{\begin{array}{lll}
0 & \text { if } & t \in] 0, t_{1}[\cup] t_{2}, L[ \\
\frac{t-t_{1}}{\delta} v & \text { if } & t \in\left[t_{1}, t_{1}+\delta\right], \\
v & \text { if } & t \in] t_{1}+\delta, t_{2}-\delta[ \\
\frac{t_{2}-t}{\delta} v & \text { if } & t \in\left[t_{2}-\delta, t_{2}\right] .
\end{array}\right.
$$

Notice that there exists $\varepsilon_{0}>0$ such that the function $\gamma+\varepsilon \varphi_{\delta}$ is bi-lipschitz for any $\left.\varepsilon \in\right] 0, \varepsilon_{0}$ [ and identifies the boundary of a set $F$ of finite perimeter such that $F \triangle E \Subset U$. By (16) we get

$$
\begin{aligned}
\int_{U}\left(\chi_{E}-\chi_{F}\right) H(x) \mathrm{d} x & \leq P(F, U)-P(E, U) \\
& =\int_{0}^{L} \psi\left(\gamma^{\prime}(t)+\varepsilon \varphi_{\delta}^{\prime}(t)\right)-\psi\left(\gamma^{\prime}(t)\right) \mathrm{d} t
\end{aligned}
$$

for any $\varepsilon \in\left(0, \varepsilon_{0}\right)$. Notice also that, by Hölder inequality,

$$
-\int_{U}\left(\chi_{E}-\chi_{F}\right) H(x) \mathrm{d} x \leq|E \triangle F|\|H\|_{L^{\infty}} \leq \varepsilon C_{1}\|H\|_{L^{\infty}}\|a-b\|
$$

for some constant $C_{1}>0$, since $E \triangle F$ is contained in an $\varepsilon$-neighbourhood of $\gamma([a, b])$.

Moreover

$$
\psi\left(\gamma^{\prime}(t)+\varepsilon \varphi_{\delta}^{\prime}(t)\right)=\psi\left(\gamma^{\prime}(t)\right)+\varepsilon\left\langle n_{\delta}(t), \varphi_{\delta}^{\prime}(t)\right\rangle+o(\varepsilon)
$$


where $n_{\delta}(t) \in \partial^{-} \psi\left(\gamma^{\prime}(t)\right) \subset X^{*}$ and $\partial^{-}$denotes the sub-differential in the sense of convex analysis. More precisely, $n_{\delta}(t)$ is such that

$$
\left\langle n_{\delta}(t), \varphi_{\delta}^{\prime}(t)\right\rangle=\max \left\{\left\langle n, \varphi_{\delta}^{\prime}(t)\right\rangle: n \in \partial^{-} \psi\left(\gamma^{\prime}(t)\right)\right\}
$$

Letting $\varepsilon \rightarrow 0^{+}$we obtain

$$
-C_{1}\|H\|_{L^{\infty}}\|a-b\| \leq \int_{0}^{L}\left\langle n_{\delta}(t), \varphi_{\delta}^{\prime}(t)\right\rangle \mathrm{d} t
$$

and

$$
\int_{0}^{L}\left\langle n_{\delta}(t), \varphi_{\delta}^{\prime}(t)\right\rangle \mathrm{d} t=\frac{1}{\delta} \int_{t_{1}}^{t_{1}+\delta}\left\langle n_{\delta}(t), v\right\rangle \mathrm{d} t-\frac{1}{\delta} \int_{t_{2}-\delta}^{t_{2}}\left\langle n_{\delta}(t), v\right\rangle \mathrm{d} t .
$$

Notice that, by (17), since $\varphi_{\delta}^{\prime}$ is either parallel or opposite to $v, n_{\delta}(t)$ can be chosen independent of $\delta$ for $t \in\left[t_{1}, t_{1}+\delta\right] \cup\left[t_{2}-\delta, t_{2}\right]$. Possibly passing to a subsequence $\delta_{n} \rightarrow 0^{+}$, we have

$$
\frac{1}{\delta_{n}} \int_{t_{1}}^{t_{1}+\delta_{n}} n_{\delta_{n}}(t) \mathrm{d} t \rightarrow n_{1}\left(t_{1}\right), \quad \frac{1}{\delta_{n}} \int_{t_{2}-\delta_{n}}^{t_{2}} n_{\delta_{n}}(t) \mathrm{d} t \rightarrow n_{2}\left(t_{2}\right),
$$

where $n_{i}\left(t_{i}\right) \in \partial^{-} \psi\left(\gamma^{\prime}\left(t_{i}\right)\right), i \in\{1,2\}$. It follows

$$
\begin{aligned}
\max \left\{\left\langle n_{1}-n_{2}, v\right\rangle: n_{1} \in \partial^{-} \psi\left(\gamma^{\prime}\left(t_{1}\right)\right), n_{2} \in \partial^{-} \psi\left(\gamma^{\prime}\left(t_{2}\right)\right)\right\} & \geq\left\langle n_{1}\left(t_{1}\right)-n_{2}\left(t_{2}\right), v\right\rangle \\
& \geq-C_{2}\|a-b\|
\end{aligned}
$$

with $C_{2}=C_{1}\|H\|_{\infty}$. If we differentiate the equality $\psi\left(\gamma^{\prime}(t)\right)=\varphi^{o}\left(R \lambda \gamma^{\prime}(t)\right)$ in Corollary 6.10 , recalling that $\partial^{-} \varphi^{o}\left(R \lambda \gamma^{\prime}(t)\right)=\lambda \nu_{E}^{*}(\gamma(t))$, we obtain

$$
\max \left\{\langle n, v\rangle: n \in \partial^{-} \psi\left(\gamma^{\prime}(t)\right)\right\}=\max \left\{\left\langle\tilde{n}, \lambda^{*-1} R \lambda v\right\rangle: \tilde{n} \in \nu_{E}^{*}(\gamma(t))\right\},
$$

for any $\left.v \in \partial B_{1}, t \in\right] 0, L[$. By (18), this implies

$$
\min \left\{\left\langle\tilde{n}_{1}-\tilde{n}_{2}, w\right\rangle: \tilde{n}_{1} \in \nu_{E}^{*}\left(\gamma\left(t_{1}\right)\right), \tilde{n}_{2} \in \nu_{E}^{*}\left(\gamma\left(t_{2}\right)\right)\right\} \leq C_{3}\|a-b\|,
$$

for any $w \in \bar{B}_{1}^{*}$, with a constant $C_{3}>0$ independent of $w$.

Given $x, y \in \partial^{*} E \cap U$, let now

$$
D(x, y):=\min \left\{\left\|\tilde{n}_{x}-\tilde{n}_{y}\right\|_{s}: \tilde{n}_{x} \in \nu_{E}^{*}(x), \tilde{n}_{y} \in \nu_{E}^{*}(y)\right\}
$$

then from (19) and from the minimax theorem (see for instance [11]) we get

$$
\begin{aligned}
D(x, y) & \leq C \min _{n \in \nu_{E}^{*}(x)-\nu_{E}^{*}(y)} \max _{w \in \bar{B}_{1}^{*}}\langle n, w\rangle \\
& =C \max _{w \in \bar{B}_{1}^{*}} \min _{n \in \nu_{E}^{*}(x)-\nu_{E}^{*}(y)}\langle n, w\rangle \leq C C_{3}\|x-y\| .
\end{aligned}
$$

We shall show that condition (20) implies that the set $\partial E \cap U$ is the graph of a lipschitz function in a neighbourhood of any point. Indeed, since $B_{1}$ is not a triangle, we get that, given three different points $\nu_{1}, \nu_{2}, \nu_{3} \in \partial B_{1}^{*}$, such that $\nu_{1}^{*} \cap \nu_{2}^{*} \neq \emptyset, \nu_{1}^{*} \cap \nu_{3}^{*} \neq \emptyset$ and $\nu_{1}^{*} \cap \nu_{2}^{*} \cap \nu_{3}^{*}=\emptyset$, there holds $\nu_{2}^{*} \cap \nu_{3}^{*}=\emptyset$, i.e. there exists a constant $c>0$ such that $\left\|v_{2}-v_{3}\right\|_{s} \geq c$ for any $v_{2} \in \nu_{2}^{*}, v_{3} \in \nu_{3}^{*}$. 


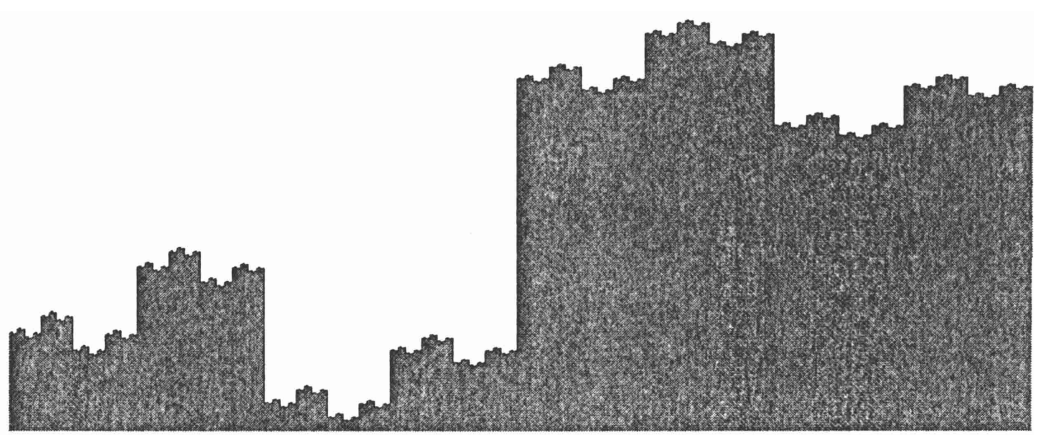

Figure 3. The set $E$ of Example 1.

Let now $x \in \partial E \cap U$ and let $x_{k} \rightarrow x, y_{k} \rightarrow x, x_{k}, y_{k} \in \partial^{*} E$. We can also assume that $\liminf _{k} \| \nu_{E}\left(x_{k}\right)-$ $\nu_{E}\left(y_{k}\right) \|>0$, otherwise this would imply that $\partial E$ is locally the graph of a lipschitz function $f: \nu^{\perp} \rightarrow \mathbb{R} v$, with $v \in \nu^{*}, \nu=\lim _{j} \nu_{E}\left(x_{k_{j}}\right)=\lim _{j} \nu_{E}\left(y_{k_{j}}\right)$, for a subsequence $x_{k_{j}}$ (resp. $\left.y_{k_{j}}\right)$ of $x_{k}$ (resp. $\left.y_{k}\right)$. Since $D\left(x_{k}, y_{k}\right) \rightarrow 0$, possibly passing to a subsequence, we can find $v \in \partial B_{1}$ and $\nu_{1}, \nu_{2} \in \partial B_{1}^{*}$ such that $\nu_{E}\left(x_{k}\right) \rightarrow \nu_{1}, \nu_{E}\left(y_{k}\right) \rightarrow \nu_{2}$, $\nu_{1} \neq \nu_{2}$ and $\nu_{1}^{*} \cap \nu_{2}^{*}=v$. We claim that, for such a $v$, we also have dist $\left(v, \nu_{E}^{*}\left(z_{k}\right)\right) \rightarrow 0$ for any sequence $z_{k} \rightarrow x$, $z_{k} \in \partial^{*} E$ (here we set $\left.\operatorname{dist}\left(v, \nu_{E}^{*}\left(z_{k}\right)\right):=\min \left\{\|v-n\|_{s}: n \in \nu_{E}^{*}\left(z_{k}\right)\right\}\right)$. In fact, if it is not true we can find (passing again to a subsequence) $\tilde{v} \in \partial B_{1}, \tilde{v} \neq v$ and $\nu_{3} \in \partial B_{1}^{*}$, such that $\nu_{E}\left(z_{k}\right) \rightarrow \nu_{3}$ and $\tilde{v}=\nu_{1}^{*} \cap \nu_{3}^{*}$. Then, since $B_{1}$ is not a triangle, there exists a constant $c>0$ such that $\left\|v_{2}-v_{3}\right\|_{s} \geq c$ for any $v_{2} \in \nu_{2}^{*}, v_{3} \in \nu_{3}^{*}$, and this contradicts the fact that $D\left(y_{k}, z_{k}\right) \rightarrow 0$. So we get $\operatorname{dist}\left(v, \nu_{E}^{*}\left(z_{k}\right)\right) \rightarrow 0$, for any sequence $z_{k} \rightarrow x$. This implies that there exist $r, c>0$ such that $\left\langle v, \nu_{E}(y)\right\rangle \geq c>0$ for any $y \in \partial^{*} E \cap B_{r}(x) \subseteq \partial^{*} E \cap U$, i.e. $\partial E$ is the graph of a lipschitz function along $v$ in the ball $B_{r}(x)$, and the first statement of the theorem is proved.

Assume that the vector $v$, defined as above, is not unique, i.e. there exists another vector $w \in \partial B_{1}, w \neq v$, such that $\operatorname{dist}\left(v, \nu_{E}^{*}\left(z_{k}\right)\right) \rightarrow 0$ for any sequence $z_{k} \rightarrow x, z_{k} \in \partial^{*} E$. This implies that $\nu_{E}^{*}\left(z_{k}\right)$ is constant in a neighbourhood of $x$, hence $\partial E$ is a segment locally around $x$. Let $x_{1}, x_{2} \in \partial E \cap \bar{U}, x_{1} \neq x \neq x_{2}$, be the extremal points of such a segment in $U$, then in $x_{i}, i \in\{1,2\}$, is uniquely defined a vector $v\left(x_{i}\right) \in \partial B_{1}$ as above (if $x_{i} \in \partial U$ we set $\left.v\left(x_{i}\right):=v\right)$. We define $v(x) \in \partial B_{1}$ as the linear interpolation between $v\left(x_{1}\right)$ and $v\left(x_{2}\right)$ in $x$. In this way we get a lipschitz function $v: \partial E \cap U \rightarrow \partial B_{1}$ such that $v(y) \in \nu_{E}(y)^{*}$ for any $y \in \partial^{*} E \cap U$.

The hypothesis that $B_{1}$ is not a triangle is technical and we believe that it is not necessary in order to get the result.

\section{Counter-examples}

Example 1. We give an example of a set $E \in \mathcal{M}_{\omega}(\Omega), \Omega \subseteq X \simeq \mathbb{R}^{2}$, which is not the graph of a lipschitz function in a subset of the boundary of positive $\mathcal{H}^{1}$-measure, even if $\omega(\rho)=o\left(\rho^{\alpha}\right)$ for any $\alpha>0$.

Let $X=\left(\mathbb{R}^{2},\|\cdot\|\right)$, where $\|(x, y)\|:=\max \{|x|,|y|\}$ for $(x, y) \in \mathbb{R}^{2}$. Let $\left\{a_{k}\right\}_{k \geq 1}$ be the sequence in $[0,1]$ defined as

$$
a_{k}:=\frac{2 j_{k}+1}{2^{n_{k}+1}}, \quad n_{k}:=\left\lfloor\ln _{2} k\right\rfloor, \quad j_{k}:=k-2^{n_{k}}
$$

so that $k=2^{n_{k}}+j_{k}$ and $0 \leq j_{k}<2^{n_{k}}$

Let also $f: \mathbb{R} \rightarrow \mathbb{R}$ be the function defined as

$$
f(x):=\sum_{k=1}^{\infty}(-1)^{n_{k}} \alpha_{k} \chi_{\left[a_{k},+\infty[\right.}(x),
$$

where $\left\{\alpha_{n}\right\}_{n \in \mathbb{N}}$ is a decreasing sequence of positive numbers such that $\sum_{n} \alpha_{n}<+\infty$. 
Notice that, if $E:=\left\{(x, y) \in \mathbb{R}^{2}: y \leq f(x)\right\}$, then $\partial E=\Gamma_{f}$ it is not the graph of a lipschitz function in any point $p=(x, y)$ such that $0<x<1$ and $x \notin \mathbb{Q}$, because any neighbourhood of $p$ contains points $\left(x^{\prime}, y^{\prime}\right)$ with $D f\left(\left\{x^{\prime}\right\}\right)>0$ and points $\left(x^{\prime \prime}, y^{\prime \prime}\right)$ with $D f\left(\left\{x^{\prime \prime}\right\}\right)<0$. Fix now a function $\omega$ satisfying the required assumptions. We will show that it is possible to find a sequence $\left\{\alpha_{n}\right\}$ such that $E$ is an $\omega$-minimum in $\left.\Omega:=\right] 0,1[\times \mathbb{R}$. Indeed, for all $\rho>0$ there exists $n(\rho):=\frac{1}{\rho}$ such that $\left|a_{i}-a_{j}\right|>2 \rho$ for all $i, j \leq n(\rho), i \neq j$. Let now $\left\{\alpha_{n}\right\}$ such that $\sum_{m \geq n(\rho)} \alpha_{m}<\rho \omega(\rho)$, for all $\rho>0$.

Let now $\rho_{0}>0$ be such that $\omega\left(\rho_{0}\right)<1$ and let $p=\left(x_{0}, y_{0}\right) \in \partial E \cap \Omega$ be such that $x_{0} \notin \mathbb{Q}$. Then, given $F$ such that $F \Delta E \Subset B_{\rho}(p)$, with $\rho<\rho_{0}$ and $\rho \in \mathbb{Q}$, we distinguish two cases:

1. $\partial E \cap \partial B_{\rho}(p)=\left\{p_{1}, p_{2}\right\}$. In this case there exists a minimal set $E_{0} \in \mathcal{M}_{0}\left(B_{\rho}(p)\right)$, such that $E_{0} \triangle E \subset B_{\rho}(p)$; for example $E_{0}$ can be obtained by taking the subgraph of the linear function between $p_{1}$ and $p_{2}$. In this case we get

$$
\begin{aligned}
P\left(E, B_{\rho}(p)\right) & \leq P\left(E_{0}, B_{\rho}(p)\right)+\sum_{m \geq n(\rho)} \alpha_{m} \\
& <P\left(F, B_{\rho}(p)\right)+\rho \omega(\rho) \leq(1+\omega(\rho)) P\left(F, B_{\rho}(p)\right) .
\end{aligned}
$$

2. $\partial E \cap \partial B_{\rho}(p)$ consists of more than two points. In this case one of the sets (and not both)

$$
I^{ \pm}:=\left\{(x, y) \in \partial B_{\rho}(p): y=y_{0} \pm 1\right\}
$$

intersects $\Gamma_{f}$ in more than one point. Let us consider the set

$$
\widetilde{B}:=\left\{(x, y) \in \mathbb{R}^{2}:\left|x-x_{0}\right|<1,\left|y-y_{0}\right|<2\right\} \supset B_{\rho}(p) .
$$

Since the condition $\omega(\rho)<1$ implies $\sum_{m \geq n(\rho)} \alpha_{m}<\rho \omega(\rho)<\rho$, we have that $\partial E \cap \partial \widetilde{B}$ consists of exactly two points. Reasoning as in the previous case and letting $D:=\widetilde{B} \backslash B_{\rho}(p)$, we get

$$
\begin{aligned}
P\left(E, B_{\rho}(p)\right) & =P(E, \widetilde{B})-P(E, \bar{D}) \\
& \leq P\left(E_{0}, \widetilde{B}\right)+\sum_{m \geq n(\rho)} \alpha_{m}-P(E, \bar{D}) \\
& <P(F, \widetilde{B})+\rho \omega(\rho)-P(F, \bar{D}) \\
& \leq(1+\omega(\rho)) P\left(F, B_{\rho}(p)\right),
\end{aligned}
$$

which gives $E \in \mathcal{M}_{\omega}(\Omega)$.

Example 2. We now provide an example of minimal set in $\mathbb{R}^{3}$ showing that Lemma 6.2 does not hold in the three-dimensional case (see also [29] for a discussion of similar examples).

Let $X=\left(\mathbb{R}^{3},\|\cdot\|\right)$, where $\|x\|:=\max \left\{\left|x_{1}\right|,\left|x_{2}\right|,\left|x_{3}\right|\right\}$ for any $x=\left(x_{1}, x_{2}, x_{3}\right) \in \mathbb{R}^{3}$. Notice that $X$ is a Banach space endowed with the norm $\|\cdot\|$. Let also

$$
E:=\left\{x=\left(x_{1}, x_{2}, x_{3}\right) \in \mathbb{R}^{3} \text { s. t. } x_{3} \leq \operatorname{sgn}\left(x_{1}\right) \operatorname{sgn}\left(x_{2}\right)\right\} .
$$


We have that $E \in \mathcal{M}_{0}$. Indeed, let us consider the vector field $n: X \rightarrow X$ given by

$$
n(x):=-\left(\operatorname{sgn}\left(x_{2}\right) e_{1}, \operatorname{sgn}\left(x_{1}\right) e_{2},-1\right) .
$$

Notice that $\operatorname{div} n=0$ in the sense of distributions, $\|n\|=1$ and the normal component of $n$ is continuous and equal to 1 across the faces of $\partial E$, i.e. $n$ provides a calibration of $E$ in $X$. By Gauss-Green Theorem, it follows that $E \in \mathcal{M}_{0}$. Indeed, let $F \subset X$ be a set of finite perimeter such that $E \Delta F \Subset B_{R}$ for some $R>0$, then

$$
\begin{aligned}
0 & =\int_{B_{R}} \operatorname{div} n * \rho_{\varepsilon}(x)\left(\chi_{E}(x)-\chi_{F}(x)\right) \mathrm{d} x=\left\langle D \chi_{E}-D \chi_{F}, n * \rho_{\varepsilon}\right\rangle \\
& \geq\left\langle D \chi_{E}\left(B_{R}\right), n * \rho_{\varepsilon}\right\rangle-P\left(F, B_{R}\right) .
\end{aligned}
$$

Since $\lim _{\varepsilon \rightarrow 0^{+}}\left\langle\nu_{E}, n * \rho_{\varepsilon}\right\rangle=1$ on the faces of $\partial E$, passing to the limit as $\varepsilon \rightarrow 0^{+}$we obtain that $P\left(F, B_{R}\right)$ $\geq P\left(E, B_{R}\right)$.

On the other hand, it is easy to check that $0 \in \partial E$ and

$$
\lim _{\rho \rightarrow \infty} \operatorname{Ecc}_{E}(0, \rho)=0, \quad \liminf _{\rho \rightarrow 0^{+}} \mathbf{E c c}_{E}(0, \rho)>0 .
$$

We thank F. Morgan for several useful discussions on this topic.

\section{REFERENCES}

[1] F.J. Almgren, Optimal isoperimetric inequalities. Indiana U. Math. J. 35 (1986) 451-547.

[2] F.J. Almgren and J. Taylor, Flat flow is motion by crystalline curvature for curves with crystalline energies. J. Diff. Geom. $42(1995)$ 1-22.

[3] F.J. Almgren, J. Taylor and L. Wang, Curvature-driven flows: A variational approach. SIAM J. Control Optim. 31 (1993) 387-437.

[4] M. Amar and G. Bellettini, A notion of total variation depending on a metric with discontinuous coefficients. Ann. Inst. H. Poincaré Anal. Non Linéaire 11 (1994) 91-133.

[5] L. Ambrosio, Corso introduttivo alla Teoria Geometrica della Misura ed alle Superfici Minime. Scuola Normale Superiore of Pisa (1997).

[6] L. Ambrosio, N. Fusco and D. Pallara, Functions of bounded variation and free discontinuity problems. Oxford U. P. (2000).

[7] L. Ambrosio, V. Caselles, S. Masnou and J.M. Morel, Connected Components of Sets of Finite Perimeter and Applications to Image Processing. J. EMS 3 (2001) 213-266.

[8] L. Ambrosio and E. Paolini, Partial regularity for the quasi minimizers of perimeter. Ricerche Mat. XLVIII (1999) 167-186.

[9] G. Bellettini, M. Paolini and S. Venturini, Some results on surface measures in Calculus of Variations. Ann. Mat. Pura Appl. 170 (1996) 329-359.

[10] E. Bombieri, Regularity theory for almost minimal currents. Arch. Rational Mech. Anal. 78 (1982) 99-130.

[11] H. Brezis, Opérateurs Maximaux Monotones. North Holland, Amsterdam (1973).

[12] Y.D. Burago and V.A. Zalgaller, Geometric inequalities. Springer-Verlag, Berlin, Grundlehren der Mathematischen Wissenschaften XIV (1988).

[13] G. David and S. Semmes, Uniform rectifiability and quasiminimizing sets of arbitrary codimension. Mem. Amer. Math. Soc. $687(2000)$.

[14] E. De Giorgi, Nuovi teoremi relativi alle misure $(r-1)$-dimensionali in uno spazio a $r$ dimensioni. Ricerche Mat. 4 (1955) 95-113.

[15] H. Federer, A note on the Gauss-Green theorem. Proc. Amer. Math. Soc. 9 (1958) 447-451.

[16] H. Federer, Geometric Measure Theory. Springer-Verlag, Berlin (1969).

[17] W.H. Fleming, Functions with generalized gradient and generalized surfaces. Ann. Mat. 44 (1957) 93-103.

[18] I. Fonseca, The Wulff theorem revisited. Proc. Roy. Soc. London 432 (1991) 125-145.

[19] I. Fonseca and S. Müller, A uniqueness proof for the Wulff theorem. Proc. Roy. Soc. Edinburgh 119 (1991) 125-136. 
[20] E. Giusti, Minimal surfaces and functions of bounded variation. Birkhäuser, Boston-Basel-Stuttgart, Monogr. in Math. 80 (1984) XII.

[21] B. Kirchheim, Rectifiable metric spaces: Local structure and regularity of the Hausdorff measure. Proc. AMS 121 (1994) 113-123.

[22] S. Luckhaus and L. Modica, The Gibbs-Thompson relation within the gradient theory of phase transitions. Arch. Rational Mech. Anal. 107 (1989) 71-83.

[23] F. Morgan, The cone over the Clifford torus in $\mathbb{R}^{4}$ is $\Phi$-minimizing. Math. Ann. 289 (1991) 341-354.

[24] F. Morgan, C. French and S. Greenleaf, Wulff clusters in $R^{2}$. J. Geom. Anal. 8 (1998) 97-115.

[25] A.P. Morse, Perfect blankets. Trans. Amer. Math. Soc. 61 (1947) 418-442.

[26] J. Taylor, Crystalline variational problems. Bull. Amer. Math. Soc. (N.S.) 84 (1978) 568-588.

[27] J. Taylor, Motion of curves by crystalline curvature, including triple junctions and boundary points. Differential Geometry, Proc. Symp. Pure Math. 54 (1993) 417-438.

[28] J. Taylor, Unique structure of solutions to a class of nonelliptic variational problems. Proc. Symp. Pure Math. 27 (1975) 419-427.

[29] J. Taylor and J.W. Cahn, Catalog of saddle shaped surfaces in crystals. Acta Metall. 34 (1986) 1-12. 